\title{
OPTIMAL PAYMENT OF MORTGAGES
}

\author{
XINFU CHEN, JOHN CHADAM, AND DEJIUN XIE
}

\begin{abstract}
This paper studies optimal strategies to terminate a mortgage with a fixed interest rate by paying the outstanding balance all at once at a time of borrower's choice. The problem is modelled as a free boundary problem under the assumption of the Vasicek's term structure and Black-Scholes theory. Here the free boundary is the optimal time where mortgage contract is to be terminated. A number of integral identities are derived and then used to design efficient numerical codes computing the free boundary. For numerical simulation, parameters for the Vasicek model are estimated via the method of maximum likelihood estimate using 40 years of data from US government bonds. The asymptotic behavior of the free boundary near infinite horizon is fully analyzed. Interpolating this infinite horizon behavior and a known near expiry behavior, two simple analytical approximation formulas for the optimal exercise boundary are proposed. Numerical evidence shows that the approximation formula is amazingly accurate; in general, its relative error is less than $1 \%$, for all time before expiry.
\end{abstract}

\section{INTRODUCTION}

In this paper, we consider a mortgage contract of fixed interest rate. We are interested in the problem of whether it is better for the mortgage borrower to terminate the mortgage by prepaying it with a lump sum and the optimal time to do so.

The mortgage contract under consideration has a duration $T$ (years) and a fixed mortgage interest rate $c\left(\right.$ year $\left.^{-1}\right)$. At any time $t$ during the term of the mortgage, the outstanding balance owed, $M(t)$, is reduced in the time period $[t, t+d t)$ by

$$
d M(t)=c M(t) d t-m d t \quad \forall t \leqslant T
$$

where $c M(t) d t$ is the interest accrued on the balance and $m d t$ is the payment resulting from a constant continuous rate of payment of $m$ (\$/year). In order that the mortgage be retired at $t=T$, the condition $M(T)=0$ applies so that

$$
M(t)=\frac{m}{c}\left\{1-e^{c(t-T)}\right\} .
$$

In this contract, the borrower is allowed to terminate the contract at any time $t(t<T)$ of his choice by paying a lump sum $M(t)$ to the contract issuer.

This decision for the borrower to terminate the contract depends on the alternate investment strategy available to him. Assume for simplicity that the borrower has sufficient funds, say from an unexpected windfall, a valuable collateral, or an arrangement from a financial institution, to pay back the outstanding balance at any time. Then at any moment while the contact is in effect, the decision of the borrower on whether to terminate the contract depends on the rate of (short term) return that an investment can yield on the financial market. In this paper, we shall

Date: September 19, 2006.

The author is partially supported by the National Science Foundation grant DMS-0203991. 
use the Vasicek model [4] for this short term market return rate, $r_{t}$, described by the stochastic differential equation

$$
d r=k(\theta-r) d t+\sigma d W_{t}
$$

where $k, \theta$, and $\sigma$ are assumed to be positive known constants and $W_{t}$ is the standard Wiener process. Here the units for $k, \theta, \sigma$, and $W_{t}$ are year ${ }^{-1}$, year ${ }^{-1}$, year ${ }^{-3 / 2}$ and year ${ }^{1 / 2}$ respectively. To mend the fact that the Vasicek model is not sufficient to describe the whole term structure, here we assume for simplicity that in this model the market price of risk has been incorporated into the drift $k(\theta-r)$; that is to say, the probability associated with the Brownian motion $\left\{W_{t}\right\}$ is the risk-neutral probability.

Intuitively if an overall market return rate is expected to be low (relative to $c$ ) for a certain amount of time, one should choose to terminate the contract early. On the other hand, if the market return rate is strictly larger than $c$ or if an overall market return rate is expected to be higher than $c$ for a certain amount of time, one should choose to defer the closing date by an investment in the market of the capital $M(t)$ less the obligatory payment of $m$ per unit time. Hence, at every moment that the contract is in effect the borrower must monitor the market return rate and decide whether to immediately close the contract. Statistically, there is an optimal strategy in making such a decision.

To find such a strategy, we introduce a function $V(r, t)$ being the (expected) value of the contract at time $t$ and current market return rate $r_{t}=r$. This value can be regarded as an asset that the contract issuer (the mortgage company) possesses, or a fair price that a buyer would offer to the contract issuer in taking over the contract, say, in an issuer's reconstructing or liquidation process. The value $V$ is calculated according to the borrower's optimal decision; i.e. the issuer is a passive player. Since the borrower can terminate the contract by paying $M(t)$ at any time $t$, we have

$$
0 \leqslant V(r, t) \leqslant M(t) \quad \forall r \in \mathbb{R}, t \leqslant T .
$$

This automatically implies that $V(r, T)=0$ for all $r$.

Now suppose $t<T$ and $V(r, t)<M(t)$. Then it is expected that the borrower will not terminate the contract in the near future, so statistically the value $V(r, t)$ should be determined by its expected value at time $t+d t$ plus the payment $m d t$ from the borrower; that is,

$$
V(r, t)=m d t+\mathbb{E}(V(r+d r, t+d t)) e^{-r d t}
$$

where $e^{-r d t}$ is the discount factor between time $t+d t$ and $t$. Assuming that $V$ is smooth in a neighborhood of $(r, t)$, we then can use the assumed distribution of $d r$ to calculate

$$
\mathbb{E}(V(r+d r, t+d t))=V(r, t)+\frac{\partial V(r, t)}{\partial t} d t+\frac{\partial V(r, t)}{\partial r}[k(\theta-r)] d t+\frac{\partial^{2} V(r, t)}{\partial r^{2}} \frac{\sigma^{2}}{2} d t .
$$

Hence, we obtain the following problem for $V$ defined for all $t \leq T$ and $r \in \mathbb{R}$ :

$$
\begin{cases}\frac{\partial V}{\partial t}+\frac{\sigma^{2}}{2} \frac{\partial^{2} V}{\partial r^{2}}+k(\theta-r) \frac{\partial V}{\partial r}+m=r V & \text { if } V(r, t)<M(t), t<T \\ 0 \leqslant V(r, t) \leqslant M(t):=\frac{m}{c}\left(1-e^{c(t-T)}\right) & \forall t \leqslant T, r \in \mathbb{R} .\end{cases}
$$


One recognizes that this is precisely the problem that would be obtained for an American style contingent claim from a Black-Scholes analysis using the Vasicek model for the underlier.

In our derivation, $V$ is an expected price. Nevertheless, if we assume that the market price of risk has been incorporated in the drift term $k(\theta-r)$, then a dynamic hedging portfolio using zero coupon bonds of various maturity can be constructed to replicate the claim. Thus the value $V$ is indeed the arbitrage-free price of the mortgage contract.

One can imagine that as $r$ increases, the value $V$ should decrease since the borrower will be less likely to close the contract and after inflation, the expected future payment at the fixed rate $c$ would translate into less value at the current time. Mathematically, we can verify this as follows. Note that $V_{r}:=\frac{\partial V}{\partial r}$ satisfies the differential equation, in the set where $V<M(t)$,

$$
\frac{\partial V_{r}}{\partial(T-t)}-\frac{\sigma^{2}}{2} \frac{\partial^{2} V_{r}}{\partial r^{2}}-k(\theta-r) \frac{\partial V_{r}}{\partial r}+(r+k) V_{r}=-V \leqslant 0 .
$$

By a comparison principle, we see that $V_{r}(r, t) \leqslant 0$ for all $r \in \mathbb{R}, t \leqslant T$. Therefore, there is a function $R(\cdot):(-\infty, T) \rightarrow[-\infty, \infty)$ such that for each $t<T$,

$$
V(r, t)<M(t) \Longleftrightarrow r>R(t) .
$$

We call $r=R(t)$ the optimal boundary of mortgage contract termination. That is,

the best strategy for the borrower is to terminate the mortgage contract at the first time that the spot market return rate $r_{t}$ is below $R(t)$.

The mathematical analysis for problem (1.1) has been completely carried out by Bian, Jiang, and Yi [1]. In particular, they proved that the problem is well-posed; namely problem (1.1) admits a unique solution which is smooth up to to free boundary $r=R(r)$. Also, the free boundary $R(\cdot)$ is a smooth function strictly increasing on $(-\infty, T)$, and has the asymptotic behavior

$$
R(t) \sim c-\sigma \bar{\kappa} \sqrt{T-t} \quad \text { as } t \nearrow T, \quad \bar{\kappa}=0.47386 \ldots
$$

In this paper we shall consider numerical aspects of this problem. In the course of this study, we shall provide an analytical solution to the infinite horizon problem and show that

$$
R(t) \sim R^{*}+\rho^{*} e^{-c(T-t)} \text { as } t \rightarrow-\infty
$$

where $R^{*}$ and $\rho^{*}$ are constants that can be easily calculated by solving an algebraic equation involving Hermit functions. Based on the existing near expiry behavior (1.3) and our new long term behavior (1.4), we provide two global approximations. For all $t \leqslant T$,

$$
\begin{aligned}
R(t) \approx R_{I}(t):= & c-\left(c-R^{*}\right) \sqrt{1-e^{-b^{*}(T-t)}}, \quad b^{*}:=\left(\frac{0.474 \sigma}{c-R^{*}}\right)^{2}, \\
R(t) \approx R_{I I}(t):= & c-\frac{0.474 \sigma}{\sqrt{2 c}} \sqrt{1-e^{-2 c(T-t)}}+\rho^{*}\left[e^{-c(T-t)}-1\right] \\
& +\left[R^{*}-c+\frac{0.474 \sigma}{\sqrt{2 c}}+\rho^{*}\right]\left[1-e^{-2 c(T-t)}\right] .
\end{aligned}
$$


We shall numerically demonstrate that these approximations are very accurate. In the special case when typically US economy parameters are used, we have

$$
\max _{t \leqslant T} \frac{\left|R(t)-R_{I}(t)\right|}{c-R^{*}} \leqslant 2 \%, \quad \frac{\max _{t \leq T}\left|R(t)-R_{\text {appI }}(t)\right|}{R(T)-R(-\infty)}<0.4 \% .
$$

The paper is organized as follows. In $\S 2$ we use the statistical procedure of Maximum Likelihood Estimation (MLE) to determine reasonable values for the parameters $k, \theta$ and $\sigma$ appearing in the Vasicek model to be used for the stochastic market rate of return. Without knowledge on the market price of risk, we can only believe with faith that these values should be in the vicinity of those values that incorporate the market price of risk. In $\S 3$, we make a series of transformations of variables to reduce problem (1.1) to a simpler version in terms of the heat equation. $\S 4$ develop integral identities that will be used in $\S 5$ to obtain fast and accurate numerical schemes based on Newton's method. Estimates for the asymptotic behavior of the termination boundary near and far from expiry of the mortgage are obtained in $\S 6$ and $\S 7$ respectively. These are combined in $\S 8$ to obtain simple global estimates. The paper concludes in $\S 9$ with some numerical experiments.

\section{Calibration of The Vasicek Model Using Mle}

In the Vasicek model [4] for the behavior of the rate of return, say a US government bond, the yield $r_{t}$ at time $t$ is treated as a Markov process, governed by the stochastic differential equation

$$
d r_{t}=k\left(\theta-r_{t}\right) d t+\sigma d W_{t}
$$

where $k, \theta$, and $\sigma$ are positive parameters and $\left\{W_{t}\right\}$ is the standard Wiener (Brownian) process.

To determine the numerical values of the parameters $k, \theta, \sigma$ in the Vasicek model, we use the method of maximum likelihood. Starting from an initial rate $r_{\tau}=x$, at a later time $t(t>\tau)$, the probability density $p$ for the rate $r_{t}$ to be equal to $y$ is given by

$$
\begin{aligned}
p(\tau, x ; t, y) & :=\frac{\text { Probability }\left(r_{\tau}=x, r_{t} \in(y, y+d y)\right)}{d y} \\
& =\sqrt{\frac{k}{\pi \sigma^{2}\left(1-e^{-2 k(t-\tau)}\right)}} \exp \left(-\frac{k\left[(y-\theta)-(x-\theta) e^{-k(t-\tau)}\right]^{2}}{\sigma^{2}\left(1-e^{-2 k(t-\tau)}\right)}\right) .
\end{aligned}
$$

Let $\left\{\left(\tau_{i}, x_{i}, t_{i}, y_{i}\right)\right\}_{i=1}^{n}$ be a collection of sample data where $x_{i}=r_{\tau_{i}}, y_{i}=r_{t_{i}}$, and $t_{i}>\tau_{i}$ for all $i$. Assume that the time increment $\Delta t:=t_{i}-\tau_{i}$ is independent of $i$ and that the intervals $\left\{\left[\tau_{i}, \tau_{i}+\Delta t\right)\right\}_{i=1}^{n}$ are non-overlapping. Using

$$
d\left(e^{-k t}\left(r_{t}-\theta\right)\right)=\sigma e^{-k t} d W_{t}
$$

we can show that $\left\{\left(y_{i}-\theta\right)-\left(x_{i}-\theta\right) e^{-k \Delta t}\right\}_{i=1}^{n}$ are i.i.d random variables. Hence, we can define the maximum likelihood function

$$
\Phi(k, \theta, \sigma):=\prod_{i=1}^{n} p\left(0, x_{i} ; \Delta t, y_{i}\right) .
$$


Consequently, the maximum likelihood estimators (MLE) for $k, \theta, \sigma$ are defined as the maximizer of the function $\Phi$. This results in the algebraic system

$$
\frac{\partial \Phi(k, \theta, \sigma)}{\partial k}=0, \quad \frac{\partial \Phi(k, \theta, \sigma)}{\partial \theta}=0, \quad \frac{\partial \Phi(k, \theta, \sigma)}{\partial \sigma}=0 .
$$

To simplify the system, we use the change of variables

$$
\begin{gathered}
b=e^{-k \Delta t}, \quad a=\sigma^{2}\left(1-\beta^{2}\right) / k, \quad \theta=\theta, \\
\Psi(a, b, \theta)=\ln (\sqrt{\pi} \Phi)=-\frac{n}{2} \ln a-\frac{1}{a} \sum_{i=1}^{n}\left[\left(y_{i}-\theta\right)-\left(x_{i}-\theta\right) b\right]^{2} .
\end{gathered}
$$

Then the system $\nabla_{k, \theta, \sigma} \Phi=(0,0,0)$ is equivalent to $\nabla_{b, \theta, a} \Psi=(0,0,0)$. This provides the algebraic system

$$
\begin{aligned}
\frac{1}{a} \sum_{i=1}^{n}\left[\left(y_{i}-\theta\right)-b\left(x_{i}-\theta\right)\right] & =0, \\
\frac{1}{a} \sum_{i=1}^{n}\left[\left(y_{i}-\theta\right)-b\left(x_{i}-\theta\right)\right]\left(x_{i}-\theta\right) & =0, \\
-\frac{n}{2 a}+\frac{1}{a^{2}} \sum_{i=1}^{n}\left[\left(y_{i}-\theta\right)-b\left(x_{i}-\theta\right)\right]^{2} & =0 .
\end{aligned}
$$

Introduce the random variables $X=\left(x_{1}, \cdots, x_{n}\right), \quad Y=\left(y_{1}, \cdots, y_{n}\right)$ and their statistics

$$
\begin{gathered}
\bar{X}=\frac{1}{n} \sum_{i=1}^{n} x_{i}, \quad \bar{Y}=\frac{1}{n} \sum_{i=1}^{n} y_{i}, \quad \operatorname{Cov}[X, Y]=\frac{1}{n-1} \sum_{i=1}^{n}\left(y_{i}-\bar{Y}\right)\left(x_{i}-\bar{X}\right), \\
\operatorname{Cov}[X, X]=\frac{1}{n-1} \sum_{i=1}^{n}\left(x_{i}-\bar{X}\right)^{2}, \quad \operatorname{Cov}[Y, Y]=\frac{1}{n-1} \sum_{i=1}^{n}\left(Y_{i}-\bar{Y}\right)^{2} .
\end{gathered}
$$

Then solving the equations for $a, b, \theta$ and transforming back to $k, \theta, \sigma$ we obtain the following statistics for the maximum likelihood estimators, also known as the MLEs,

$$
\begin{gathered}
b=\frac{\operatorname{Cov}[X, Y]}{\operatorname{Cov}[X, X]}, \quad k=-\frac{1}{\Delta t} \log b, \quad \theta=\frac{\bar{Y}-b \bar{X}}{1-b}, \\
\sigma^{2}=2 k \frac{n-1}{n} \frac{\operatorname{Cov}[Y, Y]-b^{2} \operatorname{Cov}[X, X]}{1-b^{2}}=2 k \frac{n-1}{n} \frac{\operatorname{Cov}[Y-b X, Y-b X]}{1-b^{2}} .
\end{gathered}
$$

Now suppose we have a list $\left\{\left(\tau_{i}, r_{i}\right)\right\}_{i=0}^{n}$ of rates of return $r_{i}$ at time $\tau_{i}$. Assume that all $\tau_{i+1}-\tau_{i}$ are positive and equal. Then we can take

$$
\Delta t=\tau_{i+1}-\tau_{i}, \quad X=\left(r_{0}, \cdots, r_{n-1}\right), \quad Y=\left(r_{1}, \cdots, r_{n}\right)
$$

and use these data to estimate the parameters $k, \theta$, and $\sigma$ in the Vasicek model.

The following Table (Figure 1) is a summary of the MLEs, one for a 13 week US Treasury Bill and the other for a 10 year US Treasury Note. We use three different time intervals $\Delta t=t_{i+1}-t_{i}$ : daily, weekly, and monthly. (We assume, as usual, that the weekend is approximately equivalent to a single trading day.) We calculated these data using 10, 20, 30, 40 year periods. One can see that within the same period, say from 1996 to 2006, the MLEs obtained by using the daily data, or the weekly data or the monthly data are basically the same.

Remark 2.1. The MLEs are not necessarily unbiased. Assume that $k$ and $b=e^{-k \Delta t}$ are known. Then $Z=\left\{z_{i}:=r_{\tau_{i}+\Delta t}-b r_{\tau_{i}}\right\}$ are i.i.d. random variables, normally distributed with 


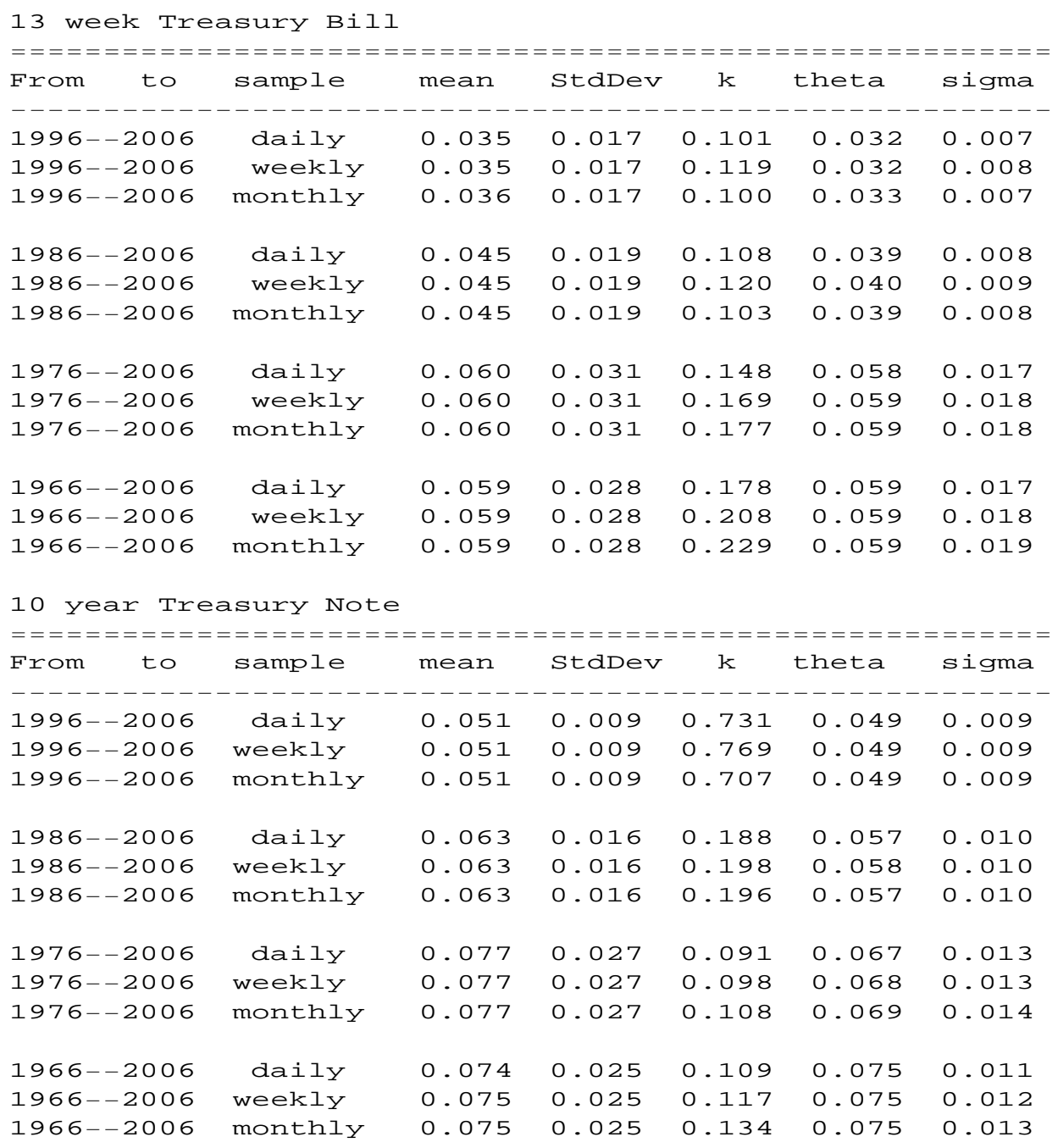

FIGURE 1. Summary of the yields for USA Government Bonds and the corresponding maximum likelihood estimators for the parameters in the Vasicek model. Where "mean" and "StdDev" represent the mean and standard deviation of the yield, measured in annual units.

mean $(1-b) \theta$ and variance $\sigma^{2}\left(1-b^{2}\right) /(2 k)$, so

$$
\begin{aligned}
\bar{\theta} & :=\frac{\bar{Z}}{1-b}=\frac{\sum_{i=1}^{n} z_{i}}{n(1-b)}:=\frac{\bar{Y}-b \bar{X}}{1-b} \\
\bar{\sigma}^{2} & :=\frac{2 k}{1-b^{2}} \operatorname{Cov}[Z, Z]=\frac{2 k}{1-b^{2}} \operatorname{Cov}[Y-b X, Y-b X]
\end{aligned}
$$

are unbiased statistics for $\theta$ and $\sigma$. This illustrates that the parameters $\sigma$ and $\theta$ in the Table are quite stable, namely, not very sensitive to the method of sampling.

To estimate $b$, note that $\left\{z_{i}:=y_{i}-b x_{i}\right\}$ being i.i.d random variables can be interpreted as saying that $b$ is the best linear indicator between $Y=\left(y_{1}, \cdots, y_{n}\right)$ and $X=\left(x_{1}, \cdots, x_{n}\right)$ so we 
have a reasonable estimator

$$
e^{-\bar{k} \Delta t}=\bar{b}=\frac{\operatorname{Cov}[Y, X]}{\operatorname{Cov}[X, X]}=1-\frac{\operatorname{Cov}[Y-X, X]}{\operatorname{Cov}[X, X]} .
$$

Here since $\tau_{i+1}=\tau_{i}+\Delta t$, i.e., $y_{i}=x_{i+1}$, the distribution of $\bar{b}$ is quite complicated. For the moment, we do not know if $\bar{b}$ is unbiased.

\section{TRANSFORMATIONS}

In this section, we shall make certain transformations to simplify the mathematical analysis of the equation for $V$; namely, we transfer the Black-Scholes equation to a heat equation. For simplicity, we shall use subscripts to denote partial derivatives.

3.1. Time to Expiry. It is convenient to use the new variables

$$
\tau:=T-t, \quad \psi(r, \tau):=\frac{c}{m}\{M(t)-V(r, t)\} .
$$

Note that $\tau$ is the time to expiry, and $\psi$ is a dimensionless quantity measuring the advantage of deferring termination, i.e., the amount of premium lost if the contract is closed at the current time $t$ and market rate $r$, in comparison to the optimal terminating strategy.

In the new variables, (1.1) is equivalent to

$$
\begin{cases}\psi_{\tau}-\frac{\sigma^{2}}{2} \psi_{r r}-k(\theta-r) \psi_{r}+r \psi=(r-c)\left(1-e^{-c \tau}\right) & \text { if } \psi(r, \tau)>0, \tau>0, \\ 0 \leqslant \psi(r, \tau) \leqslant 1-e^{-c \tau} & \forall \tau \geqslant 0, r \in \mathbb{R} .\end{cases}
$$

We remark that the constraint $\psi(r, \tau) \leqslant 1-e^{-c \tau}$ on the upper bound, which corresponds to the original constraint $V \geqslant 0$, is not needed, since one can show that $1-e^{-c \tau}$ is a super-solution so that by comparison

$$
\psi(r, \tau)<1-e^{-c \tau} \quad \forall r \in \mathbb{R}, \tau>0 .
$$

Also, differentiating in $r$ one sees that

$$
\left\{\frac{\partial}{\partial \tau}-\frac{\sigma^{2}}{2} \frac{\partial^{2}}{\partial r^{2}}-k(\theta-r) \frac{\partial}{\partial r}+(r+k)\right\} \psi_{r}=1-e^{-c \tau}-\psi \geqslant 0 \quad \text { if } \psi>0 .
$$

The maximum principle than implies that $\psi_{r}(r, t) \geqslant 0$ for all $r \in \mathbb{R}, \tau \geqslant 0$. Therefore, there exists a function $R:(-\infty, T) \rightarrow[-\infty, \infty)$ such that for each $\tau>0$,

$$
\psi(r, \tau)>0 \quad \Longleftrightarrow \quad r>R(T-\tau) .
$$

This is an equivalent way of stating (1.2). 
3.2. Dependent Variable Change. Let $h=h(r, \tau)$ be a function to be determined shortly. We make the change of variables for the unknown function $\psi$ by

$$
\phi(r, \tau):=e^{-h(r, \tau)} \psi(r, \tau) .
$$

Then the constraint for $\psi$ becomes the constraint $\phi \geqslant 0$ for $\phi$. When $\phi>0$ we have $\psi>0$ and the differential equation for $\psi$ is transformed to the following differential equation for $\phi$ :

$$
\phi_{\tau}-\frac{\sigma^{2}}{2} \phi_{r r}-\left[\sigma^{2} h_{r}+k(\theta-r)\right] \phi_{r}+q \phi=(r-c)\left(1-e^{-c \tau}\right) e^{-h}
$$

where

$$
q:=h_{\tau}-\frac{\sigma^{2}}{2} h_{r r}-h_{r}\left\{\frac{\sigma^{2}}{2} h_{r}+k(\theta-r)\right\}+r .
$$

We want to find a special $h$ such that $q \equiv 0$. To this end choose

$$
h(r, \tau)=\frac{k}{\sigma^{2}}\left(r+\frac{\sigma^{2}}{2 k^{2}}-\theta\right)^{2}+\left(k+\frac{\sigma^{2}}{2 k^{2}}-\theta\right) \tau .
$$

The equation for $\phi$ becomes

$$
\left\{\begin{array}{l}
\phi_{\tau}-\frac{\sigma^{2}}{2} \phi_{r r}-\left\{k r+\frac{\sigma^{2}}{k}-k \theta\right\} \phi_{r}=(r-c)\left(1-e^{-c \tau}\right) e^{-h} \quad \text { if } \phi>0, \\
\phi(r, \tau) \geqslant 0=\phi(r, 0) \quad \forall r \in \mathbb{R}, \tau>0 .
\end{array}\right.
$$

3.3. The Independent Variable Change. Finally, we make the change of variables

$$
x=\frac{k^{1 / 2} e^{k \tau}}{\sigma}\left[r+\frac{\sigma^{2}}{k^{2}}-\theta\right], \quad s=e^{2 k \tau}, \quad u(x, s)=\frac{2 \sqrt{\pi} k^{3 / 2}}{\sigma} \phi(r, \tau) .
$$

Then the system for $\phi$ becomes

$$
\begin{cases}u_{s}-\frac{1}{4} u_{x x}=f(x, s) & \text { if } u(x, s)>0, s>1, \\ u(x, s) \geqslant 0=u(x, 1) & \forall s>1, x \in \mathbb{R}\end{cases}
$$

where

$$
\begin{aligned}
f(x, s) & =\frac{\sqrt{\pi} k^{1 / 2}}{\sigma}(r-c)\left(1-e^{-c \tau}\right) e^{-2 k \tau-h} \\
& =\sqrt{\pi}\left(\frac{x}{\sqrt{s}}-\frac{k^{2}(c-\theta)+\sigma^{2}}{\sigma k^{3 / 2}}\right)\left(1-s^{-c /(2 k)}\right) s^{\theta /(2 k)-3 / 2-\sigma^{2} /\left(4 k^{2}\right)} e^{-\left[x / \sqrt{s}-\sigma /\left(2 k^{3 / 2}\right)\right]^{2}} .
\end{aligned}
$$

For the system (3.1) to be well-posed, it is necessary to write the system for $u=u(x, s)$ as

$$
\left\{\begin{array}{l}
\min \left\{u, \quad u_{s}-\frac{1}{4} u_{x x}-f\right\}=0 \quad \text { in } \mathbb{R} \times(1, \infty), \\
u(x, 1)=0 \quad \forall x \in \mathbb{R} .
\end{array}\right.
$$

Note that $f$ can be written as

$$
f(x, s)=\sqrt{\pi}\left(s^{\gamma}-1\right) s^{-\nu-1}(x-\beta \sqrt{s}) e^{-\left(\frac{x}{\sqrt{s}}-\alpha\right)^{2}},
$$

where $\alpha, \beta, \gamma$, and $\nu$ are dimensionless constants given by

$$
\alpha:=\frac{\sigma}{2 k^{3 / 2}}, \quad \gamma:=\frac{c}{2 k}, \quad \beta:=\frac{\sqrt{k}}{\sigma}\left(c-\theta+\frac{\sigma^{2}}{k^{2}}\right), \quad \nu:=1+\frac{\sigma^{2}}{4 k^{3}}+\frac{c-\theta}{2 k} .
$$

Once we find the free boundary $x=X(s)$ such that for each $s>1$,

$$
u(x, s)>0 \quad \Longleftrightarrow \quad x>X(s),
$$


the optimal boundary $r=R(t)$ for terminating the mortgage is given by

$$
R(t)=c+\frac{\sigma}{\sqrt{k}}\left(\frac{X(s)}{\sqrt{s}}-\beta\right\}=c+\frac{\sigma}{\sqrt{k}}\left\{\frac{X\left(e^{2 k(T-t)}\right)}{e^{k(T-t)}}-\beta\right\} .
$$

\section{Integral Equations}

4.1. Well-posedness. Using a standard theory of variational inequalities (e.g. [2]), one can show (c.f. [1]) that (3.2) admits a unique solution. In addition, there exists $X$ such that

$$
\left\{\begin{array}{l}
u_{s}-\frac{1}{4} u_{x x}=f(x, s) \mathbf{1}_{[X(s), \infty)}(x) \quad \text { in } \mathbb{R} \times(1, \infty), \\
u(x, s)>0 \quad \forall x>X(s), s>1, \\
u(x, 1)=0 \quad \forall x \in \mathbb{R}, \quad u(x, s)=0 \quad \forall s>1, x \leqslant X(s)
\end{array}\right.
$$

where

$$
\mathbf{1}_{[z, \infty)}(x)=1 \text { if } x \geqslant z, \quad \mathbf{1}_{[z, \infty)}(x)=0 \quad \text { if } x<z .
$$

Here the differential equation for $u$ is in the $L^{p}$ sense, i.e., both $u_{s}$ and $u_{x x}$ are in $L_{l o c}^{p}(\mathbb{R} \times[0, \infty))$ for any $p \in(1, \infty)$.

Denote by

$$
\Gamma(x, s):=\frac{e^{-x^{2} / s}}{\sqrt{\pi s}}
$$

the fundamental solution associated with the heat operator $\partial_{s}-\frac{1}{4} \partial_{x x}^{2}$. Using Green's identity, the solution $u$ to the differential equation in (4.1) can be expressed as

$$
u(x, s)=\int_{1}^{s} d \varsigma \int_{X(\varsigma)}^{\infty} \Gamma(x-y, s-\varsigma) f(y, \varsigma) d y \quad \forall x \in \mathbb{R}, s \geq 1 .
$$

4.2. The Integral Identities. In this section, we shall derive the following three integral identities for the unknown free boundary function $X(\cdot)$ defined on $(1, \infty)$ :

$$
\begin{aligned}
0= & \int_{1}^{s} d \varsigma \int_{X(\varsigma)}^{\infty} \Gamma(X(s)-y, s-\varsigma) f(y, \varsigma) d y=0 \quad \forall s>1, \\
0= & \int_{1}^{s} d \varsigma \int_{X(\varsigma)}^{\infty} \Gamma_{x}(X(s)-y, s-\varsigma) f(y, \varsigma) d y=0 \quad \forall s>1, \\
2 f(X(s), s)= & -\int_{1}^{s} \Gamma_{x}(X(s)-X(\varsigma), s-\varsigma) f(X(\varsigma), \varsigma) d \varsigma \\
& \quad+\int_{s}^{1} \int_{X(\varsigma)}^{\infty} \Gamma_{x}(X(s)-y, s-\varsigma) f_{y}(y, \varsigma) d y d \varsigma \quad \forall s>1 .
\end{aligned}
$$

These identities correspond to the facts $u(X(s), s)=0, u_{x}(X(s), s)=0$ and $u_{x x}(X(s)+, s)-$ $u_{x x}(X(s)-, s)=-4 f(X(s), s)$, respectively. 
4.3. The First Integral Representation. Setting $x=X(s)$ in (4.2) we immediately obtain the first integral equation (4.3) for the unknown $X(\cdot)$.

Although $u(x, s)=0$ for all $x \leqslant X(s)$, the equation (4.3) always produces the correct free boundary, as shown in the following Lemma.

Theorem 1. Suppose $X: s \in[1, \infty) \rightarrow \mathbb{R}$ is a continuous function satisfying (4.3). Define $u$ as in (4.2). Then $(X, u)$ solves (4.1) and $u$ is the unique solution to (3.2). In addition,

$$
X(s)<\beta \sqrt{s} \quad \forall s>1 .
$$

Proof. Since $X$ is continuous and $f$ is smooth and bounded, the function $u$ defined in (4.2) satisfies the differential equation in (4.1). In the domain $\{(x, s) \mid s>1, x<X(s)\}, u$ satisfies the heat equation $u_{s}=\frac{1}{4} u_{x x}$ and the zero boundary condition so $u \equiv 0$ in the domain. After transforming to the original variable $(r, \tau, \psi)$ one can show that the corresponding function $\psi$ satisfies $\psi_{r} \geqslant 0$. From this we can derive that $u>0$ when $x>X(s)$ and $s>1$. Hence, $(X, u)$ solves (4.1).

Next we prove (4.6). Let $U$ be the solution to

$$
\left\{\begin{array}{l}
U_{s}-\frac{1}{4} U_{x x}=f(x, s), \quad(x, s) \in \Omega:=\{(x, s) \mid s>1, x>\beta \sqrt{s}\}, \\
U=0 \text { on } \partial_{p} \Omega:=[\beta, \infty) \times\{0\} \cup\{(\beta \sqrt{s}, s) \mid s>1\} .
\end{array}\right.
$$

Since $f>0$ in $\Omega$, we have $U>0$ in $\Omega$ and $U_{x}(\beta \sqrt{s}, s)>0$ for all $s>1$. Comparing $u$ and $U$ on $\bar{\Omega}$ we see that $u \geqslant U$ on $\bar{\Omega}$. Since $u_{x}(X(s), s)=0$, Hopf's Lemma implies that $u>U$ when $x=\beta \sqrt{s}, s>1$. Thus, $X(s)<\beta \sqrt{s}$ for all $s>1$.

Finally, notice that $f<0$ whenever $x<\beta \sqrt{s}$, or whenever $x<X(s)$, so that $u$ satisfies the variational inequality (3.2). It is a known fact that for any given smooth bounded $f,(3.2)$ admit a unique solution; see for example, Friedman [2]. This completes the proof.

We remark that (4.3) is derived from $u(X(s), s)=0$. Since both $u_{x}(X(s), s)=0$ and $u_{s}(X(s), s)=0$, it would not be easy to find a stable and efficient scheme based solely on (4.3) and the standard Newton's method. We shall derive numerical schemes based on other integral equations for $X(\cdot)$.

4.4. The Second Integral Identity. A differentiation with respect to $x$ for $u$ in (4.2) gives, for every $x \in \mathbb{R}$ and $s \geqslant 1$,

$$
u_{x}(x, s)=\int_{1}^{s} d \varsigma \int_{X(\varsigma)}^{\infty} \Gamma_{x}(x-y, s-\varsigma) f(y, \varsigma) d y .
$$

Such differentiation is permitted since $f$ is bounded and smooth, whereas

$$
\int_{1}^{s} \int_{\mathbb{R}}\left|\Gamma_{x}(x-y, s-\varsigma)\right| d y d \varsigma=\frac{4 \sqrt{s-1}}{\sqrt{\pi}}<\infty .
$$

The condition $u_{x}(X(s), s)=0$ immediately gives us the second integral equation (4.4).

For the same reason as before, although $u_{x}(x, t)=0$ for all $x \leqslant X(s)$, a solution to (4.4) always provides us the correct answer.

Theorem 2. Suppose $X: s \in[1, \infty) \rightarrow \mathbb{R}$ is continuous and satisfies (4.4). Then it is unique and the function $u$ defined in (4.2) solves (3.2) and $(X, u)$ solves (4.1). 
The proof is analogous to that for Lemma 1 and hence is omitted.

Later we shall devise a numerical algorithm based on (4.4). For this, we need another integral identity.

4.5. The Third Integral Identity. In order to take another derivative, we use integration by parts to write

$$
u_{x}(x, s)=\int_{1}^{s}\left\{\Gamma(x-X(\varsigma), s-\varsigma) f(X(\varsigma), \varsigma)+\int_{X(\varsigma)}^{\infty} \Gamma(x-y, s-\varsigma) f_{y}(y, \varsigma) d y\right\} d \varsigma .
$$

Assume that $X(\cdot)$ is continuous. Then for $x \neq X(s)$, we can exchange the order of differentiation and integration to obtain

$$
u_{x x}(x, s)=\int_{1}^{s}\left\{\Gamma_{x}(x-X(\varsigma), s-\varsigma) f(X(\varsigma), \varsigma)+\int_{X(\varsigma)}^{\infty} \Gamma_{x}(x-y, s-\varsigma) f_{y}(y, \varsigma) d y\right\} d \varsigma .
$$

Suppose that $[X(s)-X(\varsigma)] /(s-\varsigma)^{3 / 2}$ is integrable over $\varsigma \in(1, s)$. Then

$$
\int_{1}^{s}\left|\Gamma_{x}(X(s)-X(\varsigma), s-\varsigma) f(X(\varsigma), \varsigma)\right| d \varsigma=O(1) \int_{1}^{s} \frac{|X(s)-X(\varsigma)|}{(s-\varsigma)^{3 / 2}} d \varsigma<\infty .
$$

As $f$ is smooth, we derive that

$$
\begin{aligned}
\lim _{\varepsilon \rightarrow 0+} u_{x x}(X(s) \pm \varepsilon, s)= & \mp 2 f(X(s), s)+\int_{1}^{s}\left\{\Gamma_{x}(X(s)-X(\varsigma), s-\varsigma) f(X(\varsigma), \varsigma)\right. \\
& \left.+\int_{X(\varsigma)}^{\infty} \Gamma_{x}(X(s)-y, s-\varsigma) f_{y}(y, \varsigma) d y\right\} d \varsigma .
\end{aligned}
$$

Consequently, since $u_{x x}(x, s)=0$ for all $x<X(s)$, we have $u_{x x}(X(s)+, s)=-4 f(X(s), s)$ and the integral identity $(4.5)$.

The fact that $u_{x x}(X(s)+, s)>0$ allows us to devise a stable and efficient Newton's iteration scheme to solve for $X$ from the integral equation (4.4), originated from $u_{x}(\cdot, s)=0$. As we shall see, the identity (4.5) will play an important role in simplifying our scheme.

\section{A Newton Iteration Scheme}

5.1. The Derivation. We intend to numerically solve $X$ from the integral equation (4.4). For this, we define an operator $Q$ from $\rho \in C^{1}((1, \infty))$ to $Q[\rho]$ by

$$
\begin{aligned}
Q[\rho](s) & :=\int_{s}^{1} \int_{\rho(\varsigma)}^{\infty} \Gamma_{x}(\rho(s)-y, s-\varsigma) f(y, \varsigma) d y d \varsigma \\
& =\int_{s}^{1} \int_{0}^{\infty} \Gamma_{x}(\rho(s)-\rho(\varsigma)-z, s-\varsigma) f(\rho(\varsigma)+z, \varsigma) d z d \varsigma \quad \forall s>1 .
\end{aligned}
$$

Thus, our problem is to find $X \in C([1, \infty)) \cap C^{\infty}((0, \infty))$ such that $Q[X] \equiv 0$. For this, we use Newton's method. 
To implement Newton's method, we need to calculate the first variation of $Q[\rho]$. For every smooth function $\zeta$, we compute

$$
\begin{aligned}
& Q^{\prime}[\rho, \zeta](s)=\lim _{\varepsilon \searrow 0} \frac{Q[\rho+\varepsilon \zeta](s)-Q[\rho](s)}{\varepsilon} \\
= & \int_{1}^{s} \int_{0}^{\infty}\left\{(\zeta(s)-\zeta(\varsigma)) \Gamma_{x x}(\rho(s)-\rho(\varsigma)-z, s-\varsigma) f(\rho(\varsigma)+z, \varsigma)\right. \\
& \left.+\zeta(\varsigma) \Gamma_{x}(\rho(s)-\rho(\varsigma)-z, s-\varsigma) f_{y}(\rho(\varsigma)+z, \varsigma)\right\} d z d \varsigma \\
= & \zeta(s) \int_{s}^{1}\left\{\Gamma_{x}(\rho(s)-\rho(\varsigma), s-\varsigma) f(\rho(\varsigma), \varsigma)+\int_{\rho(\varsigma)}^{\infty} \Gamma_{x}(\rho(s)-y, s-\varsigma) f_{y}(y, \varsigma) d y\right\} d \varsigma \\
& \quad-\int_{1}^{s} \zeta(\varsigma) \Gamma_{x}(\rho(s)-\rho(\varsigma), s-\varsigma) f_{y}(\rho(\varsigma), \varsigma) d \varsigma .
\end{aligned}
$$

In particular, when $\rho=X$, we can use (4.5) to simplify the expression as

$$
Q^{\prime}[X, \zeta](s)=-2 f(X(s), s) \zeta(s)-\int_{s}^{1} \zeta(\varsigma) \Gamma_{x}(X(s)-X(\varsigma), s-\varsigma) f(X(\varsigma), \varsigma) d \varsigma .
$$

Let $\Delta s$, representing a certain mesh size, be small. Suppose $\zeta \equiv 0$ on $[1, s-\Delta s]$. Then

$$
\begin{aligned}
Q^{\prime}[X, \zeta](s) & =-2 f(X(s), s) \zeta(s)-\int_{s-\Delta s}^{s} \zeta(\varsigma) \Gamma_{x}(X(s)-X(\varsigma), s-\varsigma) f(X(\varsigma), \varsigma) d \varsigma \\
& =-2 f(X(s), s) \zeta(s)+o(1)\|\zeta\|_{L^{\infty}([s-\Delta s, s])} .
\end{aligned}
$$

Here we have used the assumption that the improper integral $\int_{1}^{s}\left|\Gamma_{x}(X(s)-X(\varsigma), s-\varsigma)\right| d \varsigma$ is convergent.

5.2. The Newton Iteration. Now we use Newton's method to devise an iteration scheme for the unknown function $X$.

Suppose we have already found $X$ in $[1, s-\Delta s]$ and want to find $X$ on $(s-\Delta s, s]$. Picking an initial guess $X^{\text {old }}(s)$, say $X^{\text {old }} \equiv X(s-\Delta s)$ on $[s-\Delta s, s]$. We can find an iterative update scheme from $X^{\text {old }}$ to $X^{\text {new }}$ according the following rationale. Let $\zeta=X(s)-X^{\text {old }}(s)$ be the amount of unknown correction needed. Then $X^{\text {old }}=X-\zeta$ and using $Q[X](s)=0$ and (5.1) we have

$$
Q\left[X^{\text {old }}\right](s)=Q[X-\zeta](s)-Q[X](s) \approx 2 f(X(s), s) \zeta(s) .
$$

This gives us the approximation formula for the correction $\zeta$ in $X^{\text {new }}=X^{\text {old }}+\zeta$ :

$$
\zeta(s) \approx \frac{Q\left[X^{\text {old }}\right](s)}{2 f(X(s), s)} .
$$

Thus, we have the following Newton scheme, in a continuous setting,

$$
X^{\text {new }}(\varsigma)=X^{\text {old }}(\varsigma)+\frac{Q\left[X^{\text {old }}\right](\varsigma)}{2 f\left(X^{\text {old }}(\varsigma), \varsigma\right)} \quad \forall \varsigma \in(s-\Delta s, s] .
$$

We remark that in the interval $(1,1+\Delta s]$, one could pick the very first initial guess $X^{\text {old }} \equiv \beta$.

For theoretical analysis, we propose the following scheme for the existence of a solution $X$ to (4.4). Let $1=s_{0}<s_{1}<s_{2}<\cdots$ be mesh points in the sense that $\Delta s_{n}=s_{n}-s_{n-1}$ is not large (so that $o(1)$ in (5.1) is indeed small). Out objective is to show the existence of a solution $X$ to

$$
Q[X] \equiv 0
$$


via the following scheme: Set $X(0)=\beta$. We find iteratively the function $X$ on $\left(s_{n-1}, s_{n}\right]$, for $n=1,2, \cdots$, via the following

$$
\left\{\begin{array}{l}
X^{0}(\varsigma)=X\left(s_{n-1}\right), \quad \forall \varsigma \in\left(s_{n-1}, s_{n}\right], \quad\left(X^{0} \equiv X \quad \text { on }\left[1, s_{n-1}\right]\right) \\
X^{q+1}(\varsigma)=X^{q}(\varsigma)+\frac{Q\left[X^{q}\right](\varsigma)}{2 f\left(X^{q}(\varsigma), \varsigma\right)} \quad \forall \varsigma \in\left(s_{n-1}, s_{n}\right], q=0,1, \cdots, \\
X(\varsigma)=\lim _{q \rightarrow \infty} X^{q}(\varsigma) \quad \forall \varsigma \in\left(s_{n-1}, s_{n}\right] .
\end{array} .\right.
$$

5.3. The Operator $Q$. Since $Q[X](s)=u_{x}(X(s), s)$ involves a double integral over for $y \in$ $(X(\varsigma), \infty)$ and $\varsigma \in(1, s)$, to reduce the amount of calculation needed we shall make a simplification so that it involves only a boundary layer integral.

We begin with

$$
\begin{aligned}
u_{x}(x, s) & =\int_{s}^{1} \int_{X(\varsigma)}^{\infty} \Gamma_{x}(x-y, s-\varsigma) f(y, \varsigma) d y d \varsigma \\
& =\int_{1}^{s} \frac{\left(\varsigma^{\sigma}-1\right) d \varsigma}{s \varsigma^{\nu} \sqrt{s-\varsigma}} \int_{X(\varsigma)}^{\infty} \frac{2 s(y-x)(y-\beta \sqrt{\varsigma})}{(s-\varsigma) \varsigma} e^{-A(x, y, s, \varsigma)} d y
\end{aligned}
$$

where

$$
\begin{aligned}
A(x, y, s, \varsigma) & :=\frac{(x-y)^{2}}{s-\varsigma}+\frac{(y-\alpha \sqrt{\varsigma})^{2}}{\varsigma}=\frac{(x-\alpha \sqrt{\varsigma})^{2}}{s}+\frac{s(y-\xi)^{2}}{(s-\varsigma) \varsigma} \\
\xi=\xi(x, s, \varsigma) & :=\frac{\varsigma x+(s-\varsigma) \alpha \sqrt{\varsigma}}{s}=x-\frac{s-\varsigma}{s}(x-\alpha \sqrt{\varsigma}) .
\end{aligned}
$$

We write

$$
\begin{aligned}
& (y-x)(y-\beta \sqrt{\varsigma})=(y-\xi)(y+\xi-x-\beta \sqrt{\varsigma})+(\xi-x)(\xi-\beta \sqrt{\varsigma}) \\
= & (y-\xi)(y+\xi-x-\beta \sqrt{\varsigma})-\frac{(s-\varsigma) \varsigma}{s}\left(\frac{x}{\sqrt{\varsigma}}-\alpha\right)\left(\frac{\xi}{\sqrt{\varsigma}}-\beta\right)
\end{aligned}
$$

and use

$$
\begin{aligned}
& \frac{2 s}{(s-\varsigma) \varsigma} \int_{X(\varsigma)}^{\infty}(y-\xi)(y+\xi-x-\beta \sqrt{\varsigma}) \exp \left(-\frac{s(y-\xi)^{2}}{(s-\varsigma) \varsigma}\right) d y \\
= & \{X(\varsigma)+\xi-x-\beta \sqrt{\varsigma}\} \exp \left\{-\frac{s(X(\varsigma)-\xi)^{2}}{(s-\varsigma) \varsigma}\right\}+\int_{X(\varsigma)}^{\infty} \exp \left(-\frac{s(y-\xi)^{2}}{(s-\varsigma) \varsigma}\right) d y .
\end{aligned}
$$

Introducing the complementary error function

we can express

$$
\operatorname{Erfc}(x)=\frac{2}{\sqrt{\pi}} \int_{x}^{\infty} e^{-z^{2}} d z
$$

$$
\int_{X(\varsigma)}^{\infty} \exp \left(-\frac{s(y-\xi)^{2}}{(s-\varsigma) \varsigma}\right) d y=\frac{\sqrt{\pi}}{2} \sqrt{\frac{(s-\varsigma) \varsigma}{s}} \operatorname{Erfc}\left(\sqrt{\frac{s}{(s-\varsigma) \varsigma}}(X(\varsigma)-\xi)\right) .
$$

Thus,

$$
\begin{aligned}
& u_{x}(x, s)=\int_{1}^{s} \frac{\left(\varsigma^{\gamma}-1\right) e^{-A(x, X(\varsigma), s, \varsigma)}\{X(\varsigma)-\beta \sqrt{\varsigma}+\xi-x\}}{s \varsigma^{\nu} \sqrt{s-\varsigma}} d \varsigma \\
& +\int_{1}^{s} \frac{\sqrt{\pi}\left(\varsigma^{\gamma}-1\right) e^{-\frac{(x-\alpha \sqrt{\varsigma})^{2}}{s}}\left\{\frac{1}{2}-\left(\frac{x}{\sqrt{\varsigma}}-\alpha\right)\left(\frac{\xi}{\sqrt{\varsigma}}-\beta\right)\right\} \operatorname{Erfc}\left(\frac{\sqrt{s}(X(\varsigma)-\xi)}{\sqrt{(s-\varsigma) \varsigma}}\right)}{s^{3 / 2} \varsigma^{\nu-1 / 2}} d \varsigma
\end{aligned}
$$


Therefore,

$$
\begin{aligned}
u_{x}(x, s) & =\int_{1}^{s} \frac{G_{1}(x, X(\varsigma), s, \varsigma)}{\sqrt{s-\varsigma}} d \varsigma+\int_{1}^{s} G_{2}(x, X(\varsigma), s, \varsigma) d \varsigma \quad \forall x \in \mathbb{R}, s>1, \\
Q[X](s) & =\int_{1}^{s} G_{2}(X(s), X(\varsigma), s, \varsigma) d \varsigma-2 \int_{1}^{s} G_{1}(X(s), X(\varsigma), s, \varsigma) d \sqrt{s-\varsigma} \quad \forall s>1
\end{aligned}
$$

where

$$
\begin{aligned}
& G_{1}(x, y, s, \varsigma):=\frac{\varsigma^{\gamma}-1}{s \varsigma^{\nu}}\left\{y-\beta \sqrt{\varsigma}-\frac{s-\varsigma}{s}(x-\alpha \sqrt{\varsigma})\right\} e^{-\frac{(x-y)^{2}}{s-\varsigma}-\frac{(y-\alpha \sqrt{\varsigma})^{2}}{\varsigma}}, \\
& G_{2}(x, y, s, \varsigma):=\frac{\sqrt{\pi}\left(\varsigma^{\gamma}-1\right) e^{-(x-\alpha \sqrt{\varsigma})^{2} / s}}{s^{3 / 2} \varsigma^{\nu-1 / 2}}\left\{\frac{1}{2}-\left(\frac{x}{\sqrt{\varsigma}}-\alpha\right)\left(\frac{x \sqrt{\varsigma}}{s}+\frac{s-\varsigma}{s} \alpha-\beta\right)\right\} * \\
& \operatorname{Erfc}\left(\sqrt{\frac{s}{(s-\varsigma) \varsigma}}(y-x)+\left(\frac{x}{\sqrt{\varsigma}}-\alpha\right) \sqrt{\frac{s-\varsigma}{s}}\right), \\
& G_{1}(x, x, s, s)=\frac{\left(s^{\gamma}-1\right)(x-\beta \sqrt{s}) e^{-(x / \sqrt{s}-\alpha)^{2}}}{s^{\nu+1}}=\frac{1}{\sqrt{\pi}} f(x, s), \\
& G_{2}(x, x, s, s)=\frac{\sqrt{\pi}\left(s^{\gamma}-1\right) e^{-(x / \sqrt{s}-\alpha)^{2}}}{s^{\nu+1}}\left\{\frac{1}{2}-\left(\frac{x}{\sqrt{s}}-\alpha\right)\left(\frac{x}{\sqrt{s}}-\beta\right)\right\} .
\end{aligned}
$$

5.4. The Standard Numerical Scheme. Suppose we use mesh points

$$
1=s_{0}<s_{1}<s_{2}<\cdots .
$$

We denote by $X_{i}$ the approximation of $X\left(s_{i}\right), i=0,1,2, \cdots$. One can check that

$$
X_{0}=X\left(s_{0}\right)=X(1)=\beta .
$$

We can use the trapezoid rule to discretize the integral for $Q[X]\left(s_{n}\right)$ :

$$
\begin{aligned}
Q[X]\left(s_{n}\right) \approx & \sum_{i=1}^{n}\left(\sqrt{s_{n}-s_{i-1}}-\sqrt{s_{n}-s_{i}}\right)\left(G_{1}\left(X_{n}, X_{i}, s_{n}, s_{i}\right)+G_{1}\left(X_{n}, X_{i-1}, s_{n}, s_{i-1}\right)\right) \\
& +\sum_{i=1}^{n}\left(s_{i}-s_{i-1}\right) \frac{G_{2}\left(X_{n}, X_{i}, s_{n}, s_{i}\right)+G_{2}\left(X_{n}, X_{i-1}, s_{n}, s_{i-1}\right)}{2} .
\end{aligned}
$$

Consider $z=X_{n}$ as an unknown. Numerically, we solve for it from the equation

$$
Q_{n}(z)=0
$$

where, since $G_{1}(\cdot, \cdot, \cdot, 1) \equiv 0$ and $G_{2}(\cdot, \cdot, \cdot, 1) \equiv 0, Q_{n}(\cdot)$ is defined by, for $n=1$,

$$
Q_{1}(z):=\sqrt{s_{1}-1} G_{1}\left(z, z, s_{1}, s_{1}\right)+\frac{s_{1}-1}{2} G_{2}\left(z, z, s_{1}, s_{1}\right)
$$

and for $n \geq 2$,

$$
\begin{aligned}
Q_{n}(z):= & \sqrt{s_{n}-s_{n-1}} G_{1}\left(z, z, s_{n}, s_{n}\right)+\frac{s_{n}-s_{n-1}}{2} G_{2}\left(z, z, s_{n}, s_{n}\right) \\
& +\sum_{i=1}^{n-1}\left\{\left(\sqrt{s_{n}-s_{i-1}}-\sqrt{s_{n}-s_{i+1}}\right) G_{1}\left(z, X_{i}, s_{n}, s_{i}\right)+\frac{\left(s_{i+1}-s_{i-1}\right)}{2} G_{2}\left(z, X_{i}, s_{n}, s_{i}\right)\right\} .
\end{aligned}
$$


Suppose $X_{0}, X_{1}, \cdots, X_{n-1}$ are known. We solve for $X_{n}=z$ from $Q_{n}(z)=0$ by the following iteration:

$$
\left\{\begin{array}{l}
z_{0}=X_{n-1}+\frac{X_{n-1}-X_{n-2}}{s_{n-1}-s_{n-2}}\left(s_{n}-s_{n-1}\right) \\
z_{q+1}=z_{q}+\frac{Q_{n}\left(z_{q}\right)}{2 f\left(z_{q}, s_{n}\right)}, \quad q=0,1,2, \cdots \\
X_{n}=z_{q+1} \text { if }\left|z_{q+1}-z_{q}\right| \leq \varepsilon, \text { a given tolerance. }
\end{array}\right.
$$

Here $z_{0}$ is an initial guess derived from a linear interpolation. We point out that Newton's method is quite efficient. For instance, in the example summarized in the left table in Figure 2, when 1024 evenly distributed division points are use for the interval $\left[1, e^{2 k T}\right] \ni s$ with $T=1$ (year) and the tolerance is set to be $\varepsilon=5 \times 10^{-7}$, the sum of all the $q$ 's in the 1024 steps are 287 ; that is, the average number $q$ of iteration is about 0.3 , which means $q=0$ in most updating steps from $X_{n-1}$ to $X_{n}$.

Numerical simulation shows that this numerical scheme has error of size

$$
X\left(s_{n}\right)-X_{n}=O((\Delta s))
$$

where $\Delta s$ is the mesh size. That is to say, when the mesh size is halved, the error reduces by half.

Finally, we remark that when $n=1$, the equation for $X_{1}$ is equivalent to

$$
X_{1}-\beta \sqrt{s_{1}}=-\frac{\sqrt{\pi} \sqrt{s_{1}-1}}{2}\left\{\frac{1}{2}-\left(\frac{X_{1}}{\sqrt{s_{1}}}-\alpha\right)\left(\frac{X_{1}}{\sqrt{s_{1}}}-\beta\right)\right\} .
$$

This gives a rough approximation

$$
X_{1} \approx \beta-\frac{\sqrt{\pi}}{4} \sqrt{s_{1}-1} \approx \beta-0.443 \sqrt{s_{1}-1} .
$$

As we shall see in the next section, this approximation is close, but not very accurate. The error comes from our Trapezoid rule for singular integrals.

5.5. Upgraded Numerical Scheme. In general, one can improve the rate of convergence for numerical integration by using higher order quadrature rules. Since in the current situation singular integrals are involved, higher order quadrature rules are not very effective. Here we introduce a modified Trapezoid rule designed specifically for the singular integrals at hand.

Notice that for any constants $a<b \leqslant s$ and linear function $g(x)$ on $[a, b]$ we have

$$
\begin{aligned}
& \int_{a}^{b} \frac{g(x)}{\sqrt{s-x}} d x=\int_{a}^{b} \frac{(b-x) g(a)+(x-a) g(b)}{(b-a) \sqrt{s-x}} d x \\
= & \frac{2(b-a)}{3(\sqrt{s-a}+\sqrt{s-b})^{2}}\{[\sqrt{s-a}+2 \sqrt{s-b}] g(a)+[2 \sqrt{s-a}+\sqrt{s-b}] g(b)\} .
\end{aligned}
$$

Thus, we can use the following discretization for the function $Q[X]\left(s_{n}\right)$. When $n=1$,

$$
\bar{Q}_{1}(z)=\frac{4 \sqrt{s_{1}-1}}{3} G_{1}(z, z, s, s)+\frac{s_{1}-1}{2} G_{2}(z, z, s, s) .
$$


When $n \geq 2$,

$$
\begin{aligned}
\bar{Q}_{n}(z)= & \frac{4 \sqrt{s_{n}-s_{n-1}}}{3} G_{1}\left(z, z, s_{n}, s_{n}\right)+\frac{s_{n}-s_{n-1}}{2} G_{2}\left(z, z, s_{n}, s_{n}\right) \\
& +\sum_{i=1}^{n-1} \frac{s_{i+1}-s_{i-1}}{2} G_{2}\left(z, X_{i}, s_{n}, s_{i}\right) \\
& +\sum_{i=1}^{n-1} \frac{2 G_{1}\left(z, X_{i}, s_{n}, s_{i}\right)}{3}\left\{\frac{\left(s_{i}-s_{i-1}\right)\left(\sqrt{s_{n}-s_{i}}+2 \sqrt{s_{n}-s_{i-1}}\right)}{\left(\sqrt{s_{n}-s_{i}}+\sqrt{s_{n}-s_{i-1}}\right)^{2}}\right. \\
& \left.+\frac{\left(s_{i+1}-s_{i}\right)\left(\sqrt{s_{n}-s_{i}}+2 \sqrt{s_{n}-s_{i+1}}\right)}{\left(\sqrt{s_{n}-s_{i}}+\sqrt{s_{n}-s_{i+1}}\right)^{2}}\right\} .
\end{aligned}
$$

Setting $X_{0}=\beta$ and a "ghost" value $X_{-1}=\beta+0.334 \sqrt{s_{1}-1}$, we can calculate $\left\{X_{n}\right\}$ iteratively for $n=1,2, \cdots$ by the following scheme

$$
\left\{\begin{array}{l}
z_{0}=X_{n-1}+\frac{X_{n-1}-X_{n-2}}{s_{n-1}-s_{n-2}}\left(s_{n}-s_{n-1}\right), \\
z_{q+1}=z_{q}+\frac{\bar{Q}_{n}\left(z_{q}\right)}{2 f\left(z_{q}, s_{n}\right)}, \quad q=0,1,2, \cdots, \\
X_{n}=z_{q+1} \quad \text { if }\left|z_{q+1}-z_{q}\right| \leq \varepsilon, \text { a given tolerance. }
\end{array}\right.
$$

When $\varepsilon$ is set to be $5 \times 10^{-7}$, the average number of iteration needed is about 0.2 , i.e., in most of the calculation, $q$ in (5.4) is equal to 0 . When $\varepsilon=10^{-9}$, the average number $q$ of iteration is about 2 . The rate of convergence is observed by numerical experimentation to be about $O\left((\Delta s)^{3 / 2}\right)$ :

$$
\left.X\left(s_{n}\right)-X_{n}=O(\Delta s)^{3 / 2}\right) .
$$

That is, when the mesh size $\Delta s$ is halved, the error reduces by a factor $2 \sqrt{2}=2.8$. At some special points, we have observed convergence rate of order $(\Delta s)^{2}$.

Finally, we remark that when $n=1$, the equation for $X_{1}$ is equivalent to

$$
\frac{4}{3}\left(X_{1}-\beta \sqrt{s_{1}}\right)=-\frac{\sqrt{\pi} \sqrt{s_{1}-1}}{2}\left\{\frac{1}{2}-\left(\frac{X_{1}}{\sqrt{s_{1}}}-\alpha\right)\left(\frac{X_{1}}{\sqrt{s_{1}}}-\beta\right)\right\} .
$$

This gives a very accurate approximation

$$
X_{1} \approx \beta-\frac{3 \sqrt{\pi}}{16} \sqrt{s_{1}-1} \approx \beta-0.332 \sqrt{s_{1}-1} .
$$

As we shall see in the next section, this approximation is almost the true asymptotic expansion, which reads $X(s)=\beta-[0.334 \ldots+o(1)] \sqrt{s-1}$ as $s \searrow 1$.

5.6. A Numerical Example. The following two tables in Figure 2 illustrate the rate of convergence for uniform mesh size. In this example, we take a typical US economy in 2006: in annual units,

$$
c=0.055, \quad \theta=0.05, \quad \sigma=0.015, \quad k=0.15 .
$$

One notices that the Newton iteration converges very fast; for example, when 1024 evenly distributed grid points are used for the interval $\left[1, e^{2 k T}\right]$ with $T=1$ (year), the total number of iterations for the two schemes are 287 and 213 respectively, which means iteration is not 
needed in most updates. Also one sees that the upgraded scheme is significantly better than the standard scheme.

\begin{tabular}{rrrll}
\multicolumn{3}{c}{ Standard } & Tolerance $=$ & $5 . \times 10^{-7}$ \\
Grid & Iteration Solution & Improvement & Rate \\
8 & 61 & 0.2161798 & $3 . \times 10^{-2}$ & 3.1 \\
16 & 90 & 0.2303882 & $1.4 \times 10^{-2}$ & 2.1 \\
32 & 127 & 0.2373004 & $6.9 \times 10^{-3}$ & 2.1 \\
64 & 183 & 0.2406784 & $3.4 \times 10^{-3}$ & 2.0 \\
128 & 238 & 0.2423363 & $1.7 \times 10^{-3}$ & 2.0 \\
256 & 353 & 0.2431532 & $8.2 \times 10^{-4}$ & 2.0 \\
512 & 326 & 0.2435571 & $4 . \times 10^{-4}$ & 2.0 \\
1024 & 275 & 0.2437574 & $2 . \times 10^{-4}$ & 2.0
\end{tabular}

\begin{tabular}{|c|c|c|c|c|}
\hline \multicolumn{5}{|c|}{$\times 10^{-7}$} \\
\hline Grid & eration & Solution & mprovement & Rate \\
\hline 8 & 21 & 0.2436451 & $2.9 \times 10^{-4}$ & -0.0 \\
\hline 16 & 37 & 0.2438225 & $1.8 \times 10^{-4}$ & 1. \\
\hline 32 & 59 & 0.2439030 & $8.1 \times 10^{-5}$ & 2. \\
\hline 64 & 85 & 0.2439357 & $3.3 \times 10^{-5}$ & 2. \\
\hline 128 & 142 & 0.2439484 & $1.3 \times 10^{-5}$ & 2. \\
\hline 256 & 266 & 0.2439531 & $4.7 \times 10^{-6}$ & 2. \\
\hline 512 & 253 & 0.2439548 & $1.7 \times 10^{-6}$ & 2. \\
\hline 1024 & 213 & 0.2439555 & $6.3 \times 10^{-7}$ & 2. \\
\hline
\end{tabular}

FiguRE 2. Rate of convergence for the standard numerical scheme (left) and the upgraded scheme (right). Here "Grid" stands for the number of grids, "Iteration" is the total Newton iterations, "Solution" is the value of $X$ at $s=e^{2 k \tau}$ with $\tau=T-t=1$ (year), "Improvement" is the difference between the current solution with that in the previous row, and "Rate" is the ratio of the consecutive improvements.

The following Figure 3 illustrates the difference of the two schemes. Since the upgraded scheme treats the singularity of the integral, the improvement of the solution at the first node is profound.

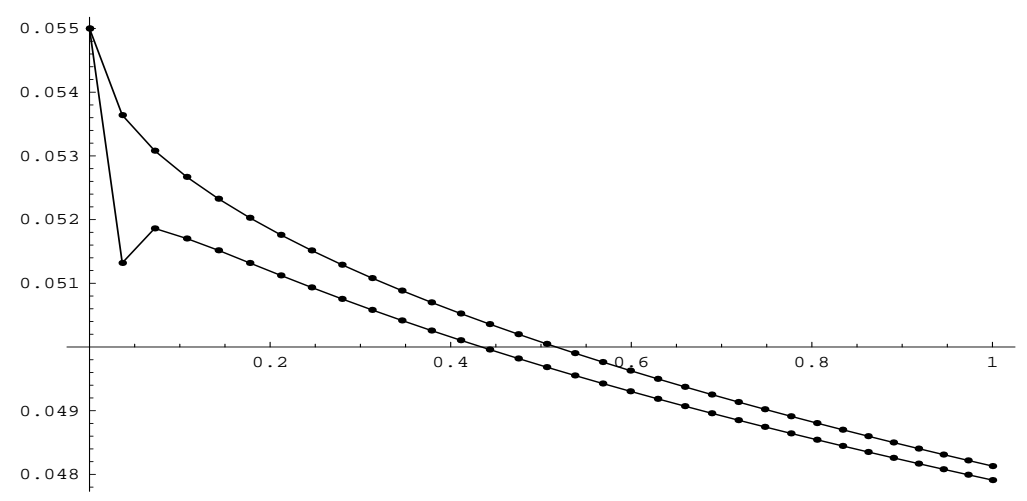

FiguRE 3. Numerical solutions of the curve $(t, R(t))$ in annual units with 32 grid points. Dots on the top curve are from the upgraded scheme; the dots on the bottom curve are for the standard scheme. The difference of the two solutions at the first node is profound.

\section{Asymptotic Behavior Near Expiry}

Here we perform a formal derivation of the asymptotic behavior of $X$ near $s=1$. A rigorous derivation using a totally different method can be found in [1]. One of the key observation here is that $f(x, s)>0$ if $x>\beta \sqrt{s}$ and $f(x, s)<0$ if $x<\beta \sqrt{s}$. 
Assume that $X(1)=\lim _{s \backslash 1} X(s)$ exists. We claim that $X(1)=\beta$. Indeed, we know that $X(s)<\beta \sqrt{s}$ for all $s>1$. Should $X(1)<\beta$, then $X(s)<\beta-\varepsilon$ for all $s \in[1,1+\varepsilon)$ for some $\varepsilon>0$. It then follows that $u$ is smooth near $(\beta-\varepsilon, 1)$, so that $u_{s s}(\beta-\varepsilon, 1)=f_{s}(\beta-\varepsilon, 1)<0$ and hence $u(\beta-\varepsilon, s)<0$ when $0<s-1 \ll 1$, contradicting the fact that $u \geq 0$. Thus, we must have $X(1)=\beta$.

Now we postulate that

$$
X(s)=\beta-\kappa \sqrt{s-1}+o(1) \sqrt{s-1} \text { as } s \searrow 1 .
$$

We then can derive the asymptotic expansion

$$
\frac{Q[X](s)}{\sigma e^{-(\beta-\alpha)^{2}}}=\int_{s}^{1}(\varsigma-1)\left\{\int_{\frac{\kappa(\sqrt{s-1}-\sqrt{\varsigma-1})}{\sqrt{s-\varsigma}}}^{\infty} e^{-z^{2}} d z-\frac{\kappa \sqrt{\varsigma-1}}{\sqrt{s-\varsigma}} e^{-\frac{\kappa^{2}(\sqrt{s-1}-\sqrt{\varsigma-1})^{2}}{s-\varsigma}}\right\} d \varsigma+o\left((s-1)^{2}\right) .
$$

Using the substitution $\varsigma=1+(s-1) t$ and sending $s \searrow 0$, we see that $\kappa$ satisfies the equation

$$
\begin{aligned}
0 & =\int_{0}^{1}\left\{\int_{\frac{\kappa(1-\sqrt{t})}{\sqrt{1-t}}}^{\infty} e^{-z^{2}} d z-\frac{\kappa \sqrt{t}}{\sqrt{1-t}} e^{-\frac{\kappa^{2}(1-\sqrt{t})}{1+\sqrt{t}}}\right\} t d t \\
& =\frac{\sqrt{\pi}}{4}-\kappa \int_{0}^{1} e^{-\frac{\kappa^{2}(1-\sqrt{t})}{1+\sqrt{t}}} \frac{(5 / 4+\sqrt{t}) t \sqrt{t}}{(1+\sqrt{t}) \sqrt{1-t}} d t
\end{aligned}
$$

After the substitution $\sqrt{t}=\frac{\kappa^{2}-z^{2}}{\kappa^{2}+z^{2}}$, the equation for $\kappa$ then becomes

$$
\sqrt{\pi}=\int_{0}^{\kappa} \frac{e^{-z^{2}}\left(\kappa^{2}-z^{2}\right)^{4}\left(18 \kappa^{2}+2 z^{2}\right)}{\left(\kappa^{2}+z^{2}\right)^{5}} d z .
$$

It is easy to see that the right-hand is an increasing function of $\kappa$, equal to 0 when $\kappa=0$ and equal to $9 \sqrt{\pi}$ when $\kappa=\infty$. Thus, there exists a unique root $\kappa$ to the equation. A numerical calculation shows that

$$
\kappa=0.3343641440309 \ldots
$$

Hence,

$$
X(s)=\beta-0.334364 \sqrt{s-1}+o(\sqrt{s-1}) \text { as } s \searrow 0 .
$$

We remark that in [1], the same asymptotic is obtained by finding the root of

$$
\int_{-\infty}^{\kappa} e^{-z^{2}} d z+e^{-\kappa^{2}} \frac{2 \kappa^{4}+4 \kappa^{2}-1}{4 \kappa^{5}+10 \kappa^{3}}=0 \Leftrightarrow \int_{\kappa}^{\infty} \frac{15 e^{-z^{2}} d z}{2 z^{4}\left(5+2 z^{2}\right)^{2}}=\sqrt{\pi} .
$$

Although it is very difficult to show analytically that the two transcendental equations (6.1) and (6.2) have the same roots, our numerical evaluation indicates that their roots are indeed identical.

Translating to the original variable, we have the asymptotic behavior:

Theorem 3. [1] Near expiry the optimal mortgage termination boundary $r=R(t)$ has the asymptotic expansion

$$
R(t) \sim c-\sigma \bar{\kappa} \sqrt{T-t} \quad \text { as } t \nearrow T, \quad \bar{\kappa}=\sqrt{2} \kappa=0.47386 \ldots
$$




\section{Asymptotic Behavior of $R(t)$ When $t \rightarrow-\infty$}

In this section, we shall prove the following

Theorem 4. There exist constants $R^{*} \in(-\infty, c)$ and $\rho^{*}>0$ such that

$$
R(t) \sim R^{*}+\rho^{*} e^{c(t-T)} \text { as } t \rightarrow-\infty .
$$

The idea here is to study first the limit $\left(R^{*}, V^{*}(\cdot)\right):=\lim _{t \rightarrow-\infty}\left(R(t), \frac{c}{m} V(\cdot, t)\right)$, which solves a so-called infinite horizon problem, and then the limit $\zeta^{*}(r):=\lim _{t \rightarrow-\infty} \zeta(r, t)$ where

$$
\zeta(r, t):=\frac{V_{t}(r, t)}{\dot{M}(t)}=-\frac{V_{t}(r, t)}{m e^{c(t-T)}} .
$$

After deriving the relation

$$
\dot{R}(t)=\frac{c \sigma^{2}}{2 m} \frac{V_{t r}(R(t)+, t)}{(c-R(t))\left(1-e^{c(t-T)}\right)}=\frac{c e^{c(t-T)} \sigma^{2}}{2} \frac{\zeta_{r}(R(t)+, t)}{(R(t)-c)\left(1-e^{c(t-T)}\right)},
$$

we see that

$$
\rho^{*}:=\frac{1}{c} \lim _{t \rightarrow-\infty} \dot{R}(t) e^{-c(t-T)}=\frac{\sigma^{2}}{2} \frac{\zeta_{r}^{*}\left(R^{*}\right)}{\left(R^{*}-c\right)} .
$$

The theorem will be proven in the following subsections. In the mean time, we shall derive formulas for $R^{*}, V^{*}(\cdot), \zeta^{*}(\cdot)$ and $\rho^{*}$.

7.1. The Infinite Horizon Problem. In [1], it is shown that $\dot{R}(t)>0$. Also, one can show that $V_{t} \leqslant 0$. Hence, there exists

$$
\lim _{t \rightarrow-\infty}\left(R(t), \frac{c}{m} V(\cdot, t)\right)=\left(R^{*}, V^{*}(\cdot)\right) .
$$

From (1.1), one derives that $\left(R^{*}, V^{*}\right)$ is a solution to the following infinite horizon problem:

$$
\begin{cases}\left\{\frac{\sigma^{2}}{2} \frac{d^{2}}{d r^{2}}+k(\theta-r) \frac{d}{d r}-r\right\} V^{*}=-c & \text { in }\left(R^{*}, \infty\right), \\ 0 \leqslant V^{*} \leqslant 1 & \text { in }\left(R^{*}, \infty\right), \\ V^{*}\left(R^{*}\right)=1, \quad V_{r}^{*}\left(R^{*}\right)=0 . & \end{cases}
$$

Theorem 5. Assume that $\sigma, k, \theta, c$ are positive constants. Then (7.2) admits a unique solution. In addition, the solution has the property that $R^{*} \in(-\infty, c)$ and $V_{r}^{*}(r)<0$ for all $r \in\left(R^{*}, \infty\right)$.

Before proving theorem 5, we first verify $(7.2)$. Let $\left(R^{*}, V^{*}\right)$ be as stated in the Theorem.

We claim that $R(t)>R^{*}$ for all $t \leqslant T$. Suppose this is not true. Then since $R(T-)=c, R$ is smooth and $\dot{R}<0$ in $(-\infty, T)$, there exists a finite $t^{*}<T$ such that $R(t)>R^{*}$ for all $t \in\left(t_{*}, T\right]$ and $R\left(t_{*}\right)=R^{*}$. We calculate, for all $r>R(t)$ and $t \in\left[t_{*}, T\right]$,

$$
\left\{\frac{\partial}{\partial(T-t)}-\frac{\sigma^{2}}{2} \frac{\partial^{2}}{\partial r^{2}}-k(\theta-r) \frac{\partial}{\partial r}+r\right\}\left(V^{*}(r) M(t)\right)=m+m e^{c(t-T)}\left[V^{*}(r)-1\right]<m
$$

since $V^{*}(r)<1$ for $r>R^{*}$. Also, $V(R(t), t)=M(t) \geq V^{*}(R(t)) M(t)$ for all $t \in\left[t_{*}, T\right]$. It then follows from a strong comparison principle for $V(r, t)$ and $V^{*}(r) M(t)$ in the set $\left\{(r, t) \mid t_{*} \leq\right.$ $t \leq T, r \geq R(t)\}$ that $M(t) V^{*}(r)<V(r, t)$ for all $r>R(t), t \in\left[t_{*}, T\right)$. In addition, as $R$ is smooth, by Hopf's Lemma, we should also have $V_{r}\left(R\left(t_{*}\right)+, t_{*}\right)>V_{r}^{*}\left(R\left(t_{*}\right)\right) M\left(t_{*}\right)=0$, which is 
impossible since we know that $M(t)=V\left(R\left(t_{*}\right), t_{*}\right) \geq V\left(r, t_{*}\right)$ for all $r$. Hence, we must have $R(t)>R^{*}$ for all $t \leqslant T$. In addition, by the comparison just established,

$$
R^{*}<R(t)<c, \quad V^{*}(r) M(t)<V(r, t)<M(t) \quad \forall t<T, r>R(t) .
$$

From these bounds and the fact that $\dot{R}>0$ and $V_{t} \leqslant 0$ we then know that there exists $(R(-\infty), V(r,-\infty)):=\lim _{t \rightarrow-\infty}(R(t), V(r, t))$. As $R(t) \geq R^{*}$, a local regularity result for parabolic equations [3] shows that $\lim _{t \rightarrow-\infty} V_{t}(r, t)=0$ for every $r>R(-\infty)$. Thus the $(R(-\infty), V(r,-\infty))$ is a solution to the infinite horizon problem (7.2). By the uniqueness result of Theorem 5, we see that (7.1) holds. We summarize the result as follows.

Lemma 7.1. Let $\left(R^{*}, V^{*}\right)$ be the unique solution to (7.2). Then both (7.1) and (7.3) hold.

In the next two subsections, we prove Theorem 5, along with formulas for $R^{*}$ and $V^{*}(\cdot)$.

7.2. The Homogeneous Equation. We begin with the homogeneous equation

$$
\left\{\frac{\sigma^{2}}{2} \frac{d^{2}}{d r^{2}}+k(\theta-r) \frac{d}{d r}-r\right\} G(r)=0, \quad r \in \mathbb{R} .
$$

In self-adjoint form, this equation can be written as

$$
\left\{e^{-k(r-\theta)^{2} / \sigma^{2}} G_{r}(r)\right\}_{r}=\frac{2}{\sigma^{2}} e^{-k(r-\theta)^{2} / \sigma^{2}} r G(r), \quad r \in \mathbb{R} .
$$

If $G_{1}$ and $G_{2}$ are two linearly independent solutions, their Wronskian satisfies

$$
G_{1 r}(r) G_{2}(r)-G_{2 r}(r) G_{1}(r)=C e^{k(r-\theta)^{2} / \sigma^{2}}
$$

where $C$ is a non-zero constant. Thus, if there is a solution bounded at $r=\infty$, it is unique up to a constant multiple. We shall now find such a solution.

Lemma 7.2. Assume that $\sigma>0$ and $k>0$. Then (7.4) admits a unique solution satisfying

$$
\lim _{r \rightarrow \infty} G(r) e^{r / k} r^{-\mu}=1, \quad \mu:=\frac{\sigma^{2}-2 \theta k^{2}}{2 k^{3}} .
$$

In addition, there exists $r_{0} \in[-\infty, 0)$ such that

$$
G_{r}<0<G \text { in }\left(r_{0}, \infty\right), \quad \int_{r_{0}}^{\infty} r e^{-k(r-\theta)^{2} / \sigma^{2}} G(r) d r=0 .
$$

In particular, (i) when $\sigma^{2} \leq 2 k^{2} \theta, r_{0}=-\infty$; (ii) when $\sigma^{2}>2 k^{2} \theta, r_{0}>-\infty$ and $G_{r}\left(r_{0}\right)=0$.

Proof. Make a change of variables

$$
x=\frac{\sqrt{k}}{\sigma}\left(r+\frac{\sigma^{2}}{k^{2}}-\theta\right), \quad H(x)=e^{r / k} G(r) .
$$

Then $H=H(x)$ satisfies the Hermite equation

$$
H_{x x}=2 x H_{x}-2 \mu H \quad \forall x \in \mathbb{R} .
$$

A particular solution of this ode is the Hermite function defined as

$$
H(\mu ; x)=\frac{(-1)^{m}}{\Gamma(m-\mu)} \int_{0}^{\infty} t^{m-\mu-1} \frac{d^{m} e^{-t^{2}-2 x t}}{d t^{m}} d t \quad \forall x, \mu \in \mathbb{C}, \quad m \in \mathbb{N} \cap(\operatorname{Re}(\mu), \infty) .
$$

Here $\Gamma(\cdot)$ is the Gamma function, $\mathbb{N}=\{0,1,2, \cdots\}$ is the set of non-negative integers, and most importantly, the integral on the right-hand side is independent of the integer $m$ and hence 
$H(\mu ; x)$ is an entire function of both variables $\mu \in \mathbb{C}$ and $x \in \mathbb{C}$. The integer $m$ here is introduced so that the integral is uniformly convergent. Without it, one can use contour integrals to express

$$
H(\mu ; x)=\frac{1}{\Gamma(-\mu)\left[1-e^{-2 \pi \mu \mathbf{i}}\right]} \int_{\omega} t^{-\mu-1} e^{-t^{2}-2 x t} d t \quad \forall x \in \mathbb{C}, \mu \in \mathbb{C} \backslash \mathbb{Z}
$$

where $\omega$ is any contour starting from $\infty e^{2 \pi \mathbf{i}}$, rotating around the origin clockwise without touching the origin and positive real axis, and finally ending at $\infty e^{0 \mathbf{i}}$. One has the relations

$$
\begin{array}{cl}
H_{x}(\mu ; x)=2 \mu H(\mu-1 ; x) & \forall x, \mu \in \mathbb{C}, \\
H(\mu+1 ; x)=2 x H(\mu ; x)-2 \mu H(\mu-1 ; x) & \forall x, \mu \in \mathbb{C}, \\
H(\mu ; x) \sim(2 x)^{\mu} \quad \text { as } x \rightarrow \infty & \forall \mu \in \mathbb{C} \\
H(\mu ; x) \sim \frac{\sqrt{\pi} e^{x^{2}} \quad \text { as } x \rightarrow-\infty}{\Gamma(-\mu)(-x)^{\mu+1} \quad} & \forall \mu \in \mathbb{C} \backslash \mathbb{N}, \\
H(\mu ;-x)=(-1)^{\mu} H(\mu ; x) & \forall x \in \mathbb{C}, \mu \in \mathbb{N} .
\end{array}
$$

Here the first two relations can be verified from the definition. For the asymptotic behavior, for every $\mu \in \mathbb{C}$, taking any $m \in \mathbb{N} \cap(\operatorname{Re}(\mu), \infty)$, we have,

$$
\begin{aligned}
\lim _{x \rightarrow \infty} \frac{H(\mu ; x)}{(2 x)^{\mu}} & =\lim _{x \rightarrow \infty} \frac{(-1)^{m}}{\Gamma(m-\mu)} \int_{0}^{\infty} s^{m-\mu-1} \frac{d^{m} e^{-s^{2} /\left(4 x^{2}\right)-s}}{d s^{m}} d s \\
& =\frac{(-1)^{m}}{\Gamma(m-\mu)} \int_{0}^{\infty} s^{m-\mu-1} \frac{d^{m} e^{-s}}{d s^{m}} d s=\frac{1}{\Gamma(m-\mu)} \int_{0}^{\infty} s^{m-\mu-1} e^{-s} d s=1
\end{aligned}
$$

by Lebesgue's Dominated Convergence theorem and the definition of the Gamma function.

When $\mu \in \mathbb{N}$, one can check that $H(\mu, x)$ is the Hermite polynomial of degree $\mu$ and that $H(\mu,-x)=(-1)^{\mu} H(\mu, x)$; in particular,

$$
\begin{gathered}
H(0 ; x)=1, \quad H(1 ; x)=2 x, \quad H(2 ; x)=4 x^{2}-2, \quad H(3 ; x)=2 x\left(4 x^{2}-6\right), \\
H(n+1 ; x)=2 x H(n ; x)-2 n H(n-1 ; x), \\
e^{-t^{2}+2 x t}=\sum_{n=0}^{\infty} \frac{H(n ; x)}{n !} t^{n} \quad \forall x, t \in \mathbb{Z} .
\end{gathered}
$$

When $\mu$ is not an integer, one can calculate

$$
\begin{aligned}
\lim _{x \rightarrow-\infty}(-x)^{\mu+1} e^{-x^{2}} H(\mu ; x) & =\lim _{x \rightarrow-\infty} \frac{1}{\Gamma(-\mu)\left[1-e^{-2 \pi \mu \mathbf{i}]}\right.} \int_{\gamma}\left(\frac{t}{-x}\right)^{-\mu-1} e^{-(t+x)^{2}} d t \\
& =\lim _{x \rightarrow-\infty} \frac{1}{\Gamma(-\mu)\left[1-e^{-2 \pi \mu \mathbf{i}]}\right.} \int_{x+\gamma}\left[1+\frac{s}{-x}\right]^{-\mu-1} e^{-s^{2}} d s \\
& =\frac{1}{\Gamma(-\mu)} \int_{-\infty}^{\infty} e^{-s^{2}} d s=\frac{\sqrt{\pi}}{\Gamma(-\mu)} .
\end{aligned}
$$

When $\mu$ is a negative integer, one can use (7.6) with $k=0$ to derive the same limit.

Finally, from $\left[e^{-x^{2}} H_{x}\right]_{x}=-2 \mu e^{-x^{2}} H$, one can derive that on the real axis, $H(\mu ; \cdot)>0>$ $H_{x}(\mu ; \cdot)$ when $\mu \leq 0$ and $H(\mu ; \cdot)$ changes sign when $\mu>0$.

Now going back to the original variable, we find that

$$
G(r) e^{-k(r-\theta)^{2} / \sigma^{2}}=e^{-x^{2}+x \sigma k^{-3 / 2}-\theta / k} H(\mu ; x) .
$$


It follows that

$$
\lim _{|r| \rightarrow \infty}\left\{\left|G_{r}(r)\right|+|G(r)|\right\} e^{-k(r-\theta)^{2} / \sigma^{2}} e^{|r| /(2 k)}=0 .
$$

Integrating (7.4) over $\mathbb{R}$ we obtain $\int_{\mathbb{R}} r e^{-k(r-\theta)^{2} / \sigma^{2}} G(r) d r=0$, where the improper integer is uniformly convergent. Finally, we have the following:

(1) When $\sigma^{2}>2 k^{2} \theta$, we have $\mu>0$. As $H(\mu ; \cdot)$ changes sign, so does $G(\cdot)$. Thus, there exists a finite real $r_{0}$ such that $G_{r}\left(r_{0}\right)=0$ and $G_{r}<0$ in $\left(r_{0}, \infty\right)$. This implies that $G>0$ in $\left[r_{0}, \infty\right)$. After integrating (7.4) over $\left[r_{0}, \infty\right)$ we obtain the integral identity in (7.5).

(2) When $\sigma^{2} \leq 2 k^{2} \theta$, we have $\mu \leq 0$, so that $H(\mu ; x)>0$ for all $x \in \mathbb{R}$. Thus, $G>0$ in $\mathbb{R}$. As $\left[e^{-k(r-\theta)^{2} / \sigma^{2}}\right]_{r}$ is positive in $(0, \infty)$ and negative in $(-\infty, 0)$, in view of $(7.7)$, we derive $G_{r}<0$ on $\mathbb{R}$. Hence (7.5) holds with $r_{0}=-\infty$. This completes the proof.

7.3. Proof of Theorem 5. We divide the proof into several steps. Suppose $\left(R^{*}, V^{*}\right)$ solves (7.2). We first establish certain properties of $\left(R^{*}, V^{*}\right)$ and then derive a formula for it, thereby obtaining both existence and uniqueness.

1. First we show that $V_{r}^{*}<0$ in $\left(R^{*}, \infty\right)$.

Suppose otherwise. Then $V_{r}^{*}\left(r_{1}\right) \geqslant 0$ at some $r_{1}>R^{*}$. Since $V^{*}\left(R^{*}\right)=1$ is a global maximum, $r_{2}:=\sup \left\{r \in\left(R^{*}, r_{1}\right) \mid V_{r}^{*}(r)<0\right\}$ is well-defined and by continuity $V_{r}^{*}\left(r_{2}\right)=0$. The case $V_{r r}^{*}\left(r_{2}\right)<0$ is impossible since it would imply $V_{r}^{*}>0$ in $\left(r_{2}-\varepsilon, r_{2}\right)$ for some small positive $\varepsilon$, contradicting the definition of $r_{2}$. The case $V_{r r}^{*}\left(r_{2}\right)=0$ is also impossible since it would imply by the ode for $V^{*}$ that $r_{2} V^{*}\left(r_{2}\right)=c>0$ and $\frac{\sigma^{2}}{2} V_{r r r}^{*}\left(r_{2}\right)=V^{*}\left(r_{2}\right)>0$ so that $V_{r}^{*}>0$ in $\left(r_{2}-\varepsilon, r\right)$ for some small positive $\varepsilon$. Hence $V_{r r}^{*}\left(r_{2}\right)>0$ and, by the ode, $r_{2} V^{*}\left(r_{2}\right)>c$. Set $r_{3}=\sup \left\{r>r_{2} \mid V_{r}^{*}>0\right.$ in $\left.\left(r_{2}, r\right)\right\}$. Then for every $r \in\left(r_{2}, r_{3}\right), r V^{*}(r)>r_{2} V\left(r_{2}\right)>c$ and $\left[e^{-k(r-\theta)^{2} / \sigma^{2}} V_{r}(r)\right]_{r}=(r V-c) e^{-k(r-\theta)^{2} / \sigma^{2}}>0$. That is, $e^{-k(r-\theta)^{2} / \sigma^{2}} V_{r}$ is a strictly increasing function on $\left[r_{2}, r_{3}\right)$. This implies $r_{3}=\infty$ and $\lim _{r \rightarrow \infty} e^{-k(r-\theta)^{2} / \sigma^{2}} V_{r}>0$, which further implies $\lim _{r \rightarrow \infty} V_{r}^{*}=\infty$, contradicting the boundedness of $V^{*}$. Thus we must have $V_{r}^{*}<0$ in $\left(R^{*}, \infty\right)$. Consequently, $0<V^{*}<1$ in $\left(R^{*}, \infty\right)$.

2. Next we show that $R^{*}>r_{0}$. For this, consider the weighted Wronskian

$$
W(r)=\left\{V_{r}^{*}(r) G(r)-V^{*}(r) G_{r}(r)\right\} e^{-k(r-\theta)^{2} / \sigma^{2}} .
$$

It satisfies $\frac{\sigma^{2}}{2} W_{r}=-c e^{-k(r-\theta)^{2} / \sigma^{2}} G$. Integrating this equation over $(r, \infty)$ gives

$$
W(r)=\frac{2 c}{\sigma^{2}} \int_{r}^{\infty} G e^{-k(r-\theta)^{2} / \sigma^{2}} d t \quad \forall r \geqslant R^{*} .
$$

First consider the case $r_{0}>-\infty$. Should $R \leqslant r_{0}$, we would have, since $G_{r}\left(r_{0}\right)=0$ and $G>0$ on $\left[r_{0}, \infty\right)$, that $0<W\left(r_{0}\right)=V_{r}^{*}\left(r_{0}\right) G\left(r_{0}\right) e^{-k(r-\theta)^{2} / \sigma^{2}} \leqslant 0$, a contradiction.

Next, we consider the case $r_{0}=-\infty$. Then $G>0$ on $\mathbb{R}$. Should $R^{*}=-\infty$, the boundedness of $V^{*}$ implies that along a sequence $R_{j} \rightarrow-\infty, V_{r}\left(R_{j}\right) \rightarrow 0$ so that, in view of (7.7),W $\rightarrow 0$ along the sequence $\left\{R_{j}\right\}$, contradicting (7.8). Thus, we must have $R^{*}>r_{0}$.

3. Now we show that $R^{*}$ needs to satisfy the following solvability condition for $R^{*}$ :

$$
\int_{R^{*}}^{\infty}(r-c) G(r) e^{-k(r-\theta)^{2} / \sigma^{2}} d r=0, \quad R^{*}>r_{0} .
$$


In fact, substituting $V^{*}\left(R^{*}\right)=1$ and $V_{r}^{*}\left(R^{*}\right)=0$ into (7.8) at $r=r^{*}$ gives

$$
e^{-k(r-\theta)^{2} / \sigma^{2}} G_{r}\left(R^{*}\right)=-\frac{2 c}{\sigma^{2}} \int_{R^{*}}^{\infty} G e^{-k(r-\theta)^{2} / \sigma^{2}} d t .
$$

The equation in (7.9) then follows by noting that

$$
e^{-k(r-\theta)^{2} / \sigma^{2}} G_{r}\left(R^{*}\right)=\int_{\infty}^{R^{*}}\left[e^{-k(r-\theta)^{2} / \sigma^{2}} G_{r}(r)\right]_{r} d r=-\frac{2}{\omega^{2}} \int_{R^{*}}^{\infty} r e^{-k(r-\theta)^{2} / \sigma^{2}} G(r) d r .
$$

4. Here we show that (7.9) has a unique solution $R^{*}$. Since $\int_{r_{0}}^{\infty} r G(r) e^{-k(r-\theta)^{2} / \sigma^{2}} d r=0$ and $G>0$ on $\left[r_{0}, \infty\right)$, we see that $r_{0}<0$ and that the function

$$
\Psi(c, r):=\int_{r}^{\infty}(t-c) G(t) e^{-k(t-\theta)^{2} / \sigma^{2}} d t, \quad c>0, r \in \mathbb{R}
$$

has the property

$$
\Psi(c, \infty)=0, \quad \Psi_{r}(c, \cdot)<0 \text { in }(c, \infty), \quad \Psi_{r}(c, \cdot)>0 \text { in }\left(r_{0}, c\right), \quad \Phi\left(r_{0}\right)<0 .
$$

It then follows that the algebraic equation $\Psi(c, \cdot)=0$ has a unique root in $\left(r_{0}, \infty\right)$. Thus, $R^{*}$ is the unique root to (7.9) and

$$
r_{0}<R^{*}<c, \quad \lim _{c \searrow 0} R^{*}=r_{0} \in[-\infty, 0) .
$$

One notices that $\Psi(c, r)>0$ for all $r>R^{*}$.

5. We are ready now to derive a formula for $V^{*}$. Integrating over $\left[R^{*}, r\right)$ the equation (7.8) multiplied by $e^{-k(r-\theta)^{2} / \sigma^{2}} G^{-2}$ and using $V^{*}\left(R^{*}\right)=1$ we obtain

$$
V^{*}(r):=G(r)\left\{\frac{1}{G\left(R^{*}\right)}+\frac{2 c}{\sigma^{2}} \int_{R^{*}}^{r} \frac{e^{k(t-\theta)^{2} / \sigma^{2}}}{G^{2}(t)} \int_{t}^{\infty} G(s) e^{-k(s-\theta)^{2} / \sigma^{2}} d s\right\} .
$$

Using

$$
\frac{1}{G\left(R^{*}\right)}-\frac{1}{G(r)}=\int_{R^{*}}^{r} \frac{G_{r}(t)}{G^{2}(t)} d t=-\frac{2}{\sigma^{2}} \int_{R^{*}}^{r} \frac{e^{k(t-\theta)^{2} / \sigma^{2}}}{G^{2}(t)} \int_{t}^{\infty} s G(s) e^{-k(s-\theta)^{2} / \sigma^{2}} d s
$$

we can write the above expression as

$$
V^{*}(r)=1-\frac{2 G(r)}{\sigma^{2}} \int_{R^{*}}^{r} \frac{e^{k(t-\theta)^{2} / \sigma^{2}}}{G^{2}(t)} \int_{t}^{\infty}(s-c) G(s) e^{-k(s-\theta)^{2} / \sigma^{2}} d s .
$$

In conclusion, if $\left(R^{*}, V^{*}\right)$ solves $(7.2)$, then $R^{*}$ is the unique root to (7.9) and $V^{*}$ is given by (7.10), which is equivalent to (7.11).

6. Finally, from (7.10), we see that $V^{*}>0$ on $\left[R^{*}, \infty\right)$. Also, as $\Phi(c, r)>0$ for all $r>R^{*}$, we see from (7.11) that $V^{*}<1$ in $\left(R^{*}, \infty\right)$ and that $V^{*}\left(R^{*}\right)=1, V_{r}\left(R^{*}\right)=0$. It is then an easy exercise to show that $V^{*}$ in (7.10) satisfies the ode in (7.2). Thus, $\left(R^{*}, V^{*}\right)$ obtained in this manner is indeed a solution to (7.2). We hence have established the existence of a unique solution to (7.2), thereby completing the proof of Theorem 5 . 
7.4. A Parametric Relation Between $R^{*}$ and $c$. As a function of $c, R^{*}$ defined in (7.9) is monotonic and the inverse function can be written as

$$
c=\frac{\int_{R^{*}}^{\infty} r e^{-k(r-\theta)^{2} / \sigma^{2}} G(r) d r}{\int_{R^{*}}^{\infty} e^{-k(r-\theta)^{2} / \sigma^{2}} G(r) d r}, \quad R^{*} \in\left(r_{0}, \infty\right) .
$$

In terms of the Hermite function, the relation between $R^{*}$ and $c$ can be written in a parametric form with parameter $x^{*}$ by

$$
\left\{\begin{array}{l}
R^{*}=\theta-\frac{\sigma^{2}}{k^{2}}+\frac{\sigma}{\sqrt{k}} x^{*}, \\
c=\theta-\frac{\sigma^{2}}{k^{2}}+\frac{\sigma}{\sqrt{k}} \frac{\int_{x^{*}}^{\infty} y H(\mu ; y) e^{-y^{2}+a y} d y}{\int_{x^{*}}^{\infty} H(\mu ; y) e^{-y^{2}+a y} d y},
\end{array} \quad x_{*} \in\left(x_{0}, \infty\right),\right.
$$

or

$$
\left\{\begin{array}{l}
R^{*}=c+\frac{\sigma}{\sqrt{k}}\left(x^{*}-\beta\right), \quad x^{*} \in\left(x_{0}, \infty\right), \\
c=\frac{k}{2} \frac{e^{a x^{*}-x^{*}}\left\{a H\left(\mu, x^{*}\right)-H_{x}\left(\mu ; x^{*}\right)\right\}}{\int_{x^{*}}^{\infty} e^{a y-y^{2} H(\mu ; y) d y},} \quad x_{*} \in\left(x_{0}, \infty\right),
\end{array}\right.
$$

where

$$
\begin{aligned}
a & :=\frac{\sigma}{k \sqrt{k}}, \quad \mu:=\frac{\sigma^{2}-2 k^{2} \theta}{2 k^{3}}, \quad \beta=\frac{\sqrt{k}}{\sigma}\left(c+\frac{\sigma^{2}}{k^{2}}-\theta\right) \\
x_{0} & :=\inf \left\{x \mid H_{y}(\mu ; y)<a H(\mu ; y) \quad \forall y \in(x, \infty)\right\} .
\end{aligned}
$$

Here $c$ in (7.14) is obtained from $c$ in (7.13) by integrating the identity

$$
\frac{\sigma}{\sqrt{k}} y H(\mu ; y) e^{a y-y^{2}}=\frac{k}{2} \frac{d}{d y}\left\{e^{a y-y^{2}}\left[H_{y}-a H\right]\right\}+\left\{\frac{\sigma^{2}}{k^{2}}-\theta\right\} H e^{a y-y^{2}} .
$$

7.5. Asymptotic Behavior of $R(t)$ as $t \rightarrow \infty$. Recall that [1]

$$
V_{r}(R(t), t)=0, \quad V_{t}(R(t), t)=\dot{M}(t)=-m e^{c(t-T)} .
$$

This implies, by the pde for $V$ in $(1.1)$ and by differentiating $V_{r}(R(t), t)=0$ that

$$
\begin{aligned}
V_{r r}(R(t)+, t) & =\frac{2}{\sigma^{2}}\{r M(t)-m-\dot{M}\}=\frac{2 m}{c \sigma^{2}}(r-c)\left(1-e^{c(T-t)}\right), \\
\dot{R}(t) & =-\frac{V_{r t}(R(t)+, t)}{V_{r r}(R(t)+, t)}=\frac{c \sigma^{2}}{2 m} \frac{V_{t r}(R(t)+, t)}{(c-R(t))\left(1-e^{c(T-t)}\right)} .
\end{aligned}
$$

Hence, to find the asymptotic behavior of $\dot{R}(t)$ as $t \rightarrow-\infty$, it suffices to find the asymptotic behavior of $V_{t r}(R(t)+, t)$ as $t \rightarrow-\infty$. For this, we consider the function $V_{t}$, whose boundary value at $r=R(t)$ is known to be $V_{t}=\dot{M}(t)=-m e^{c(t-T)}$. Also $V_{t}$ satisfies

$$
\left\{\frac{\partial}{\partial t}+\frac{\sigma^{2}}{2} \frac{\partial^{2}}{\partial r^{2}}+k(\theta-r) \frac{\partial}{\partial r}-r\right\} V_{t}=0, \quad r>R(t), t<T .
$$

For the leading order expansion of $V_{t}$ as $t \rightarrow-\infty$, it is natural to consider

$$
\zeta(r, t):=\frac{V_{t}(r, t)}{\dot{M}(t)}=-\frac{V_{t}}{m e^{c(t-T)}} .
$$


Then $\zeta$ satisfies the following problem:

$$
\left\{\begin{array}{l}
\left\{\frac{\partial}{\partial t}+\frac{\sigma^{2}}{2} \frac{\partial^{2}}{\partial r^{2}}+k(\theta-r) \frac{\partial}{\partial r}+(c-r)\right\} \zeta(r, t)=0, \quad r>R(t), t<T, \\
\zeta(r, t)=1 \quad \forall r \leqslant R(t), t<T, \quad \zeta(r, T)=1 \quad \forall r \in \mathbb{R} .
\end{array}\right.
$$

Here the initial and boundary data for $\zeta$ follows from the fact that $V(r, t)=M(t)$ for all $r \leqslant R(t)$ and that $V(\cdot, T)=0$. We shall prove in a subsequent subsection that there is a limit

$$
\lim _{t \rightarrow-\infty} \zeta(r, t)=\zeta^{*}(r) \quad \forall r>R^{*}
$$

which satisfies the ode problem

$$
\left\{\begin{array}{l}
\left\{\frac{\sigma^{2}}{2} \frac{d^{2}}{d r^{2}}+k(\theta-r) \frac{d}{d r}+(c-r)\right\} \zeta^{*}(r)=0 \quad \forall r>R^{*} \\
\zeta^{*}\left(R^{*}\right)=1, \quad \sup _{r \geqslant R^{*}} \zeta^{*}(r)<\infty .
\end{array}\right.
$$

For this, we have the following:

Lemma 7.3. Problem (7.17) has a unique solution, and the solution satisfies

$$
\zeta_{r}^{*}(r)<0, \quad 0<\zeta^{*}(r) \leqslant 1 \quad \forall r \geqslant R^{*} .
$$

In addition, in terms of the Hermite function, it is given by

$$
\begin{gathered}
\zeta^{*}(r)=\frac{e^{\left(R^{*}-r\right) / k} H(\mu+c / k ; x)}{H\left(\mu+c / k ; x^{*}\right)}, \\
x:=\frac{\sqrt{k}}{\sigma}\left(r+\frac{\sigma^{2}}{k^{2}}-\theta\right), \quad x^{*}:=\frac{\sqrt{k}}{\sigma}\left(R^{*}+\frac{\sigma^{2}}{k^{2}}-\theta\right), \quad \mu:=\frac{\sigma^{2}-2 k^{2} \theta}{2 k^{3}} .
\end{gathered}
$$

Now we can calculate

$$
\begin{aligned}
\lim _{t \rightarrow-\infty} \dot{R}(t) e^{-c(t-T)} & =\frac{c \sigma^{2}}{2} \lim _{t \rightarrow-\infty} \frac{\zeta_{r}(R(t)+, t)}{(R(t)-c)\left(1-e^{c(t-T)}\right)} \\
& =\frac{c \sigma^{2} \zeta_{r}^{*}\left(R^{*}\right)}{2\left(R^{*}-c\right)}=\frac{c \sigma \sqrt{k}}{2\left(c-R^{*}\right)}\left\{\frac{\sigma}{k \sqrt{k}}-\frac{H_{x}\left(\mu+c / k ; x^{*}\right)}{H\left(\mu+c / k ; x^{*}\right)}\right\} .
\end{aligned}
$$

Consequently, using

$$
R(t)=R^{*}+\int_{-\infty}^{t}\left\{\dot{R}(\hat{t}) e^{-c(\hat{t}-T)}\right\} e^{c(\hat{t}-T)} d \hat{t}
$$

we obtain the asymptotic expansion $R(t) \sim R^{*}+\rho^{*} e^{c(t-T)}$ for large negative $t$, as stated in Theorem 4, where

$$
\rho^{*}:=\frac{\sigma^{2} \zeta_{r}^{*}\left(R^{*}\right)}{2\left(R^{*}-c\right)}=\frac{\sigma \sqrt{k}}{2\left(c-R^{*}\right)}\left\{\frac{\sigma}{k \sqrt{k}}-\frac{H_{x}\left(\mu+c / k ; x^{*}\right)}{H\left(\mu+c / k ; x^{*}\right)}\right\} .
$$

Now to complete the proof of Theorem 4, it remains to prove Lemma 7.3 and (7.16), which will be the subject of the next two subsections. 
7.6. The Problem (7.17). The ode in (7.17) is homogeneous and has two linearly independent solutions, at least one of which is unbounded near $r=\infty$ (by using the Wronskian). Hence, if (7.17) has a solution, it is unique. Consider

$$
\hat{G}(r)=e^{-r / \theta} H(\mu+c / \theta ; x) .
$$

It satisfies

$$
\left\{\frac{\sigma^{2}}{2} \frac{d^{2}}{d r^{2}}+k(\theta-r) \frac{d}{d r}+(c-r)\right\} \hat{G}=0 \quad \forall r \in \mathbb{R}, \quad \hat{G}(\infty)=0 .
$$

We shall show that $\hat{G}_{r}<0$ on $\left[R^{*}, \infty\right)$. For this notice that $V^{*}$ satisfies

$$
\left\{\frac{\sigma^{2}}{2} \frac{d^{2}}{d r^{2}}+k(\theta-r) \frac{d}{d r}+(c-r)\right\} V^{*}=c\left(V^{*}-1\right)<0 \quad \forall r>R^{*} .
$$

Thus, the Wronskian of $\hat{G}$ and $V^{*}$ satisfies

$$
\frac{d}{d r}\left\{e^{-k(r-\theta)^{2} / \sigma^{2}}\left[V_{r}^{*} \hat{G}-\hat{G}_{r} V^{*}\right]\right\}=c\left(V^{*}-1\right) \hat{G} e^{-k(r-\theta)^{2} / \sigma^{2}} .
$$

Suppose that $\hat{G}_{r}<0$ on $\left[R^{*}, \infty\right)$ is not true. Then there exists $r_{1} \geq R^{*}$ such that $\hat{G}_{r}\left(r_{1}\right)=0$ and $\hat{G}_{r}<0$ on $\left(r_{1}, \infty\right)$. However, this would imply $\hat{G}>0$ on $\left[r_{1}, \infty\right)$ and that, since $V_{r}^{*}\left(r_{1}\right) \leq 0$,

$$
\begin{aligned}
0 & \geqslant V_{r}^{*} \hat{G}-\left.\hat{G}_{r} V^{*}\right|_{r=r_{1}} \\
& =e^{k\left(r_{1}-\theta\right)^{2} / \sigma^{2}} \int_{r_{1}}^{\infty} c\left(1-V^{*}\right) \hat{G} e^{-k(r-\theta)^{2} / \sigma^{2}} d r>0,
\end{aligned}
$$

a contradiction. Thus, $\hat{G}_{r}<0$ on $\left[R^{*}, \infty\right)$. Consequently, $0<\hat{G}(r)<\hat{G}\left(R^{*}\right)$ for all $r>R^{*}$ and

$$
\zeta^{*}(r)=\frac{\hat{G}(r)}{\hat{G}\left(R^{*}\right)}=\frac{e^{\left(R^{*}-r\right) / k} H(\mu+c / k ; x)}{H\left(\mu+c / k ; x^{*}\right)},
$$

is the unique solution to (7.17). This completes the proof of Lemma 7.3.

7.7. The Limit of $\zeta$ as $t \rightarrow-\infty$. Here we verify (7.16).

1. Since $V(r, t)=M(t)$ for all $r \leqslant R(t)$, we have $V_{t}(r, t)=M_{t}(t)$ for all $r \leqslant R(t)$. Also since $V(\cdot, T)=0$, we know from the pde in (1.1) that $V_{t}(r, T)=-m$ for all $r>c=R(0)$. Thus,

$$
\zeta(r, t)=1 \quad \forall r \leqslant R(t), t \leqslant T, \quad \zeta(r, T)=1 \quad \forall r \in \mathbb{R} .
$$

In addition, 0 is a subsolution and $e^{\left(c-R^{*}\right)(T-t)}$ is a super solution to $\zeta$ so that

$$
0<\zeta(r, t)<e^{\left(c-R^{*}\right)(T-t)} \quad \forall r \geqslant R^{*}, t<T .
$$

This implies that for each $t \leqslant T, \zeta(\cdot, t)$ is a bounded function.

2. Let $\zeta^{*}$ be the unique solution to (7.17), stated in Lemma 7.3. Now using $\zeta(R(t), t)=1>$ $\zeta^{*}(R(t))$ for all $t \leqslant T$ and comparing the function $\zeta$ and $\zeta^{*}$ on $\{(r, t) \mid r \geq R(t), t \leq T\}$ we see that $\zeta(r, t)>\zeta^{*}(r)$ for all $r \geqslant R(t)$. As $\zeta(r, t)=1>\zeta^{*}(r)$ for $r \in\left(R^{*}, R(t)\right]$, we see that

$$
\zeta(r, t)>\zeta^{*}(r) \quad \forall r>R^{*}, t \leqslant T .
$$

3. To estimate the upper bound, let

$$
G_{1}(r)=\zeta^{*}(r)\left\{1+\int_{R^{*}}^{r} \frac{e^{k(t-\theta)^{2} / \sigma^{2}}}{\zeta^{* 2}(t)} d t\right\} \quad \forall r \in \mathbb{R} .
$$


This is another solution to the ode in (7.17) and satisfies $\lim _{r \rightarrow \infty} G_{1}(r)=\infty$. Define

$$
\delta(t):=\inf \left\{\delta>0 \mid \zeta(r, t) \leq \zeta^{*}(r)+\delta G_{1}(r) \quad \forall r \geq R^{*}\right\}, \quad \forall t \leqslant T .
$$

Since $\zeta(\cdot, t)$ is bounded and $G_{1}(\infty)=\infty, \delta(t)$ is positive and finite. In addition,

$$
\zeta(r, t) \leqslant \zeta^{*}(r)+\delta(t) G_{1}(r) \quad \forall r \geqslant R^{*}, t \leqslant T .
$$

Furthermore, since $\dot{R}>0$, we have $\zeta(r)+\left.\delta(\hat{t}) G_{1}(r)\right|_{r=R(t)} \geq \zeta(R(t), \hat{t})=1$ for all $t<\hat{t}$. Hence, comparing $\zeta(r, t)$ and $\zeta^{*}(r)+\delta(\hat{t}) G_{1}(r)$ on $\{(r, t) \mid r \geqslant R(t), t \leqslant \hat{t}\}$ we have

$$
\zeta(r, t)<\zeta^{*}(r)+\delta(\hat{t}) G_{1}(r) \quad \forall r>R(t), t<\hat{t} \leqslant T .
$$

Hence, $0<\delta(t)<\delta(\hat{t})$ for all $t<\hat{t}_{1} \leqslant T$. Consequently, there exists

$$
\delta_{*}:=\lim _{t \rightarrow-\infty} \delta(t) \in[0, \infty) .
$$

4. Here we show that $\delta_{*}=0$. Suppose on the contrary that $\delta_{*}>0$.

(a) On the spatially bounded domain $\left\{(r, t) \mid r \in\left[R^{*}, c+2\right], t<T\right\}$, let $\hat{\zeta}$ be the solution to the boundary value problem

$$
\left\{\begin{array}{l}
\left\{\frac{\partial}{\partial t}+\frac{\sigma^{2}}{2} \frac{\partial^{2}}{\partial r^{2}}+k(\theta-r) \frac{\partial}{\partial r}+(c-r)\right\} \hat{\zeta}(r, t)=0, \quad r \in(R(t), c+2), t<T, \\
\hat{\zeta}(c+2, t)=\zeta^{*}(c+2)+\delta(t) G_{1}(c+2) \quad \forall t<T \\
\hat{\zeta}(r, T)=1 \quad \forall r \in\left[R^{*}, c+2\right], \quad \hat{\zeta}(r, t)=1 \quad \forall r \in\left[R^{*}, R(t)\right], t \leqslant T .
\end{array}\right.
$$

By comparison,

$$
\zeta(r, t) \leqslant \hat{\zeta}(r, t) \quad \forall r \in\left[R^{*}, c+2\right], \quad t \leqslant T .
$$

Also, using an elementary pde analysis, say the Fourier series, one can show that uniformly in $r \in\left[R^{*}, c+2\right], \lim _{t \rightarrow-\infty} \hat{\zeta}(r, t)=\hat{\zeta}(r,-\infty)$ where $\hat{\zeta}(r,-\infty)$ is the solution to the ode in (7.17) on $\left[R^{*}, c+2\right]$ with the boundary value

$$
\hat{\zeta}\left(R^{*},-\infty\right)=1, \quad \hat{\zeta}(c+2,-\infty)=\zeta^{*}(c+2)+\delta_{*} G_{1}(c+2) .
$$

By comparison, it is easy to see that $\hat{\zeta}(r,-\infty)<\zeta^{*}(r)+\delta_{*} G_{1}(r)$ for all $r \in\left[R^{*}, c+2\right)$. Thus, there exists $\delta_{1} \in\left(0, \delta^{*}\right)$ such that

$$
\hat{\zeta}(r,-\infty)<\zeta^{*}(r)+\delta_{1} G_{1}(r) \quad \forall r \in\left[R_{*}, c+1\right] .
$$

This also implies that there exists $t_{1} \ll-1$ such that

$$
\zeta(r, t)<\zeta^{*}(r)+\delta_{1} G_{1}(r) \quad \forall r \in\left[R_{*}, c+1\right], t \leqslant t_{1} .
$$

(b) Now we compare the function $\zeta(r, t)$ and $\zeta^{*}(r)+\delta_{1} G_{1}(r)$ on $[c+1, \infty) \times\left(-\infty, t_{1}\right]$. Since $\delta_{1}<\delta_{*}$, for each fixed $t<t_{1}$, we see from the definition of $\delta_{*}$ that the maximum of

$$
\varphi(r, t):=\zeta(r, t)-\left[\zeta^{*}(r)+\delta_{1} G_{1}(r)\right], \quad r \in\left[R^{*}, \infty\right)
$$

is positive. As $\varphi(r, t)<0$ for all $r \in\left[R^{*}, c+1\right]$ and $\varphi(\infty, t)=-\infty$, there exists $\hat{r}(t) \in(c+1, \infty)$ such that $0<\varphi(\hat{r}, t)=\max _{r \geq R^{*}} \varphi(r, t)$. Using $\varphi_{r}(\hat{r}, t)=0 \geq \varphi_{r r}(\hat{r}, t)$ and the pde for $\varphi$ we have

$$
\begin{aligned}
0 & =\varphi_{t}+\frac{\sigma^{2}}{2} \varphi_{r r}+(\eta-\theta r) \varphi_{r}+\left.(c-r) \varphi\right|_{r=\hat{r}} \\
& \leq \varphi_{t}(\hat{r}, t)+(c-\hat{r}) \varphi(\hat{r}, t)
\end{aligned}
$$


Hence, denoting $K(t):=\varphi(\hat{r}, t)=\max _{r>R(t)} \varphi(r, t)$, we have

$$
\begin{aligned}
\frac{d}{d t} K(t) & :=\liminf _{h \rightarrow 0} \frac{K(t+h)-K(t)}{h} \geq \lim _{h \rightarrow 0} \frac{\varphi(\hat{r}, t+h)-\varphi(\hat{r}, t)}{h}=\varphi_{t}(\hat{r}, t) \\
& \geq(\hat{r}-c) \varphi(\hat{r}, t) \geq \varphi(\hat{r}, t)=K(t) .
\end{aligned}
$$

Thus $\frac{d}{d t}\left[K(t) e^{-t}\right] \geqslant 0$ for all $t<t_{1}$. After integration, this gives

$$
0<K(t) \leq K\left(t_{1}\right) e^{t-t_{1}} \quad \forall t<t_{1}, \quad \lim _{t \rightarrow-\infty} K(t)=0 .
$$

This implies that for all sufficiently large negative $t, \max _{r} \geqslant R^{*} \varphi(r, t)=K(t) \leq \frac{1}{2}\left(\delta_{*}-\delta_{1}\right) \min _{r \geq R^{*}} G_{1}(r)$, so that $\zeta(r, t) \leq \zeta^{*}(r)+\frac{1}{2}\left(\delta_{1}+\delta_{*}\right) G_{1}(r)$ for all $r \geq R^{*}$ and sufficiently large negative $t$, contradicting the definition of $\delta_{*}$.

In conclusion, we must have $0=\delta^{*}=\lim _{t \rightarrow-\infty} \delta(t)$.

5. Denote $K_{1}(t)=\max _{r \in\left[R^{*}, c+2\right]}\left|\zeta(r, t)-\zeta^{*}(r)\right|$. Then

$$
0 \leqslant \lim _{t \rightarrow-\infty} K_{1}(t) \leq \sup _{r \in\left[R^{*}, c+2\right]} G_{1}(r) \lim _{t \rightarrow-\infty} \delta(t)=0 .
$$

Set

$$
K_{2}(t):=\sup _{r \geqslant c+1}\left|\zeta(r, t)-\zeta^{*}\right|=\lim _{\varepsilon \searrow 0} \max _{r \geqslant c+1}\left[\zeta(r, t)-\zeta^{*}(r)-\varepsilon G_{1}(r)\right] .
$$

Using a similar idea as in $4(\mathrm{~b})$ one can show that

$$
K_{2}(t) \leqslant K_{1}(t)+K_{2}(T) e^{(t-T)} \quad \forall t \leqslant T
$$

This implies that $\lim _{t \rightarrow-\infty} K_{2}(t)=0$. Thus,

$$
\lim _{t \rightarrow-\infty} \sup _{r \geqslant R^{*}}\left|\zeta(r, t)-\zeta^{*}(r)\right|=0
$$

Finally since $\dot{R}$ is bounded in $(-\infty, T-1]$ (c.f.[1]), one can use a local regularity theory for parabolic equations to show show that $\lim _{r \rightarrow-\infty} \zeta_{r}(R(t)+, t)=\zeta_{r}\left(R^{*}+\right)$. This completes the proof of (7.16) and also the proof of Theorem 4.

\section{Global Approximations}

Let $\tau=T-t$. So far we have discussed the behavior of $R(T-\tau)$ for small and large $\tau$ :

$$
R(T-\tau) \sim \begin{cases}c-\bar{\kappa} \sigma \sqrt{2 \tau} & \text { when } 0 \leqslant \tau \ll 1, \\ R^{*}+\rho^{*} e^{-c \tau} & \text { when } \tau \gg 1 .\end{cases}
$$

We can combine both to obtain a unified approximation that is valid for both small and large $\tau$, and hopefully, for all $\tau$. Here we propose two approximations. 
8.1. The Simple Global Approximation. We seek a simple approximation formula for $R(T-$ $\tau$ ) such that (i) it has asymptotic expansion $c+\bar{\kappa} \sigma \sqrt{\tau}$ for small positive $\tau$ and (ii) it exponentially approaches $R^{*}$ for large $\tau$. For this, we seek an approximation of the form

$$
R_{I}(T-\tau):=c-\bar{\kappa} \sigma \sqrt{\frac{1-e^{-b \tau}}{b}} .
$$

For any $b>0$, this approximation has the right asymptotic behavior for small $\tau$. To make it match with the large $\tau$ behavior, we need

$$
R^{*}=c-\sigma \bar{\kappa} \sqrt{\frac{1}{b}} \Longleftrightarrow b=\left(\frac{\bar{\kappa} \sigma}{c-R^{*}}\right)^{2}
$$

Hence, we have the first approximation for $R$ :

$$
R_{I}(T-\tau):=c-\left(c-R^{*}\right) \sqrt{1-e^{-b^{*} \tau}}, \quad b^{*}:=\left(\frac{0.474 \sigma}{c-R^{*}}\right)^{2} .
$$

Numerical evidence shows that when $\theta$ is close to $c$, the approximation is very accurate. In Figure 4 , the relative error is as small as 2 percent:

$$
\frac{\max _{t<T}\left|R(t)-R_{I}(t)\right|}{c-R^{*}} \approx 0.02 .
$$

8.2. An Enhanced Approximation. In the above approximation, we only used the information $R \sim R_{*}$ as $t \rightarrow-\infty$. Here we use the more detailed information that $R \sim R_{*}+\rho^{*} e^{-c \tau}=$ $O\left(e^{-2 c \tau}\right)$ for $t \rightarrow-\infty$ to guess the approximation $R(t) \approx R_{I I}(t)$ where

$$
R_{I I}(T-\tau):=c-\frac{0.474 \sigma \sqrt{1-e^{-2 c \tau}}}{\sqrt{2 c}}+\rho_{*}\left(e^{-c \tau}-e^{-2 c \tau}\right)+\left[R_{*}-c+\frac{0.474 \sigma}{\sqrt{2 c}}\right]\left(1-e^{-2 c \tau}\right) .
$$

It is a pleasant surprise to find in Figure 4 that for a typical parameter set, the relative error of the second approximation is reduced to 4 per thousand:

$$
\frac{\max _{t<T}\left|R(t)-R_{I I}(t)\right|}{c-R_{*}} \approx 0.004
$$

\section{Numerical Examples}

Here we display a few numerical results.

In Figure 5 we display the optimal termination boundary as one of the parameter changes, keeping others fixed. From the figure, one can reasonably conclude that the optimal mortgage termination boundary $r_{t}=R(c, \theta, k, \sigma ; t)$ is

(1) increasing in $c$,

(2) decreasing in $\theta$,

(3) increasing in $k$,

(4) decreasing in $\sigma$. 


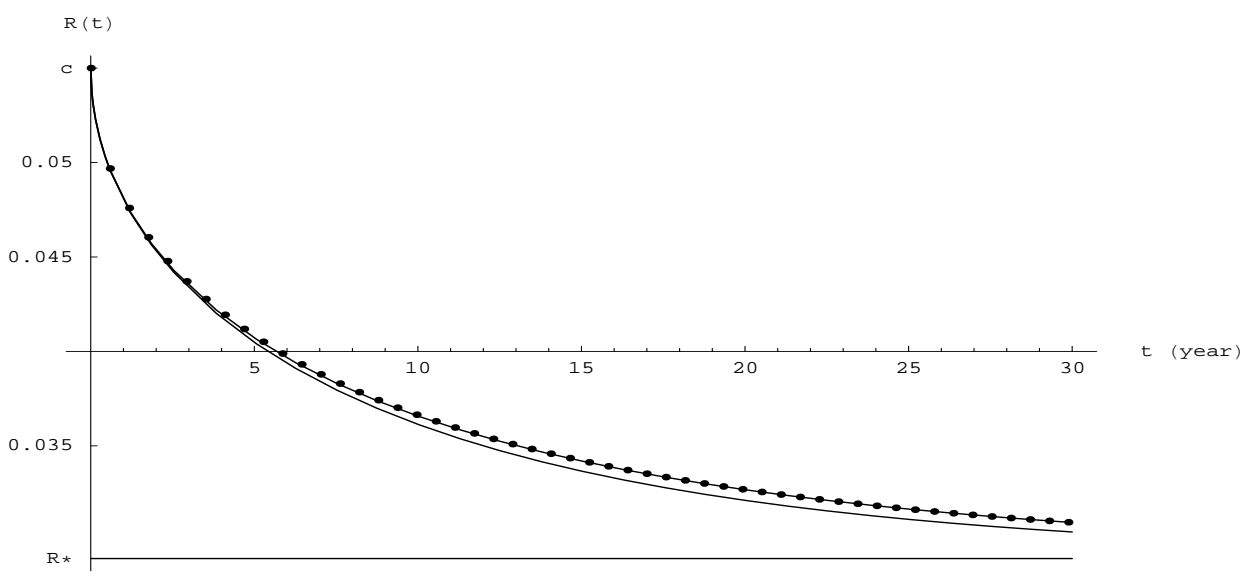

FiguRE 4. Dots represent the "true" solution, calculated by using 2048 division points in the upgraded scheme outlines in $\S 5$. The curve "on the dots" is the second approximation with maximum relative error 0.004 which is not discernable from the plot. The curve below the dots is the first approximation, with relative error 0.02. In this example, the following typical data from the US economy is used:

$\begin{aligned} c & =0.055, \quad \theta=0.050, \quad \sigma \\ \text { For this example, } R^{*} & =0.029, \quad \rho_{*}=0.0086 .\end{aligned}$

In Figure 6 we display the relative errors of our two analytical approximation formulas for the optimal boundary, as one of the parameter changes and others are kept fixed. One can see that when $0<\theta \leqslant c$, both approximations are extremely accurate.

\section{REFERENCES}

1. L. Jiang, B. Bian \& F. Yi. A Parabolic Variational Inequality Arising from the Valuation of Fixed Rate Mortgage.

2. A. Friedman, Variantional Principles and Free Boundary Problems,

3. O.A. Ladyzenskaya, V.A. Solonnikov \& N.N. Uralt́zeva, LineAR AND QUASILINEAR EQUATIONS Of PARABOLIC TYPE, Transl. Math. Mono. Vol 23, Amer. Math. Soc., Providence, RI, 1968.

4. O.A. Vasicek, An equilibrium characterization of the term structure, J. Fin. Econ, 5 (1977), 177-188.

Department of Mathematics, University of Pittsburgh, Pittsburgh, PA 15260, xinfu+@pitt.edu 

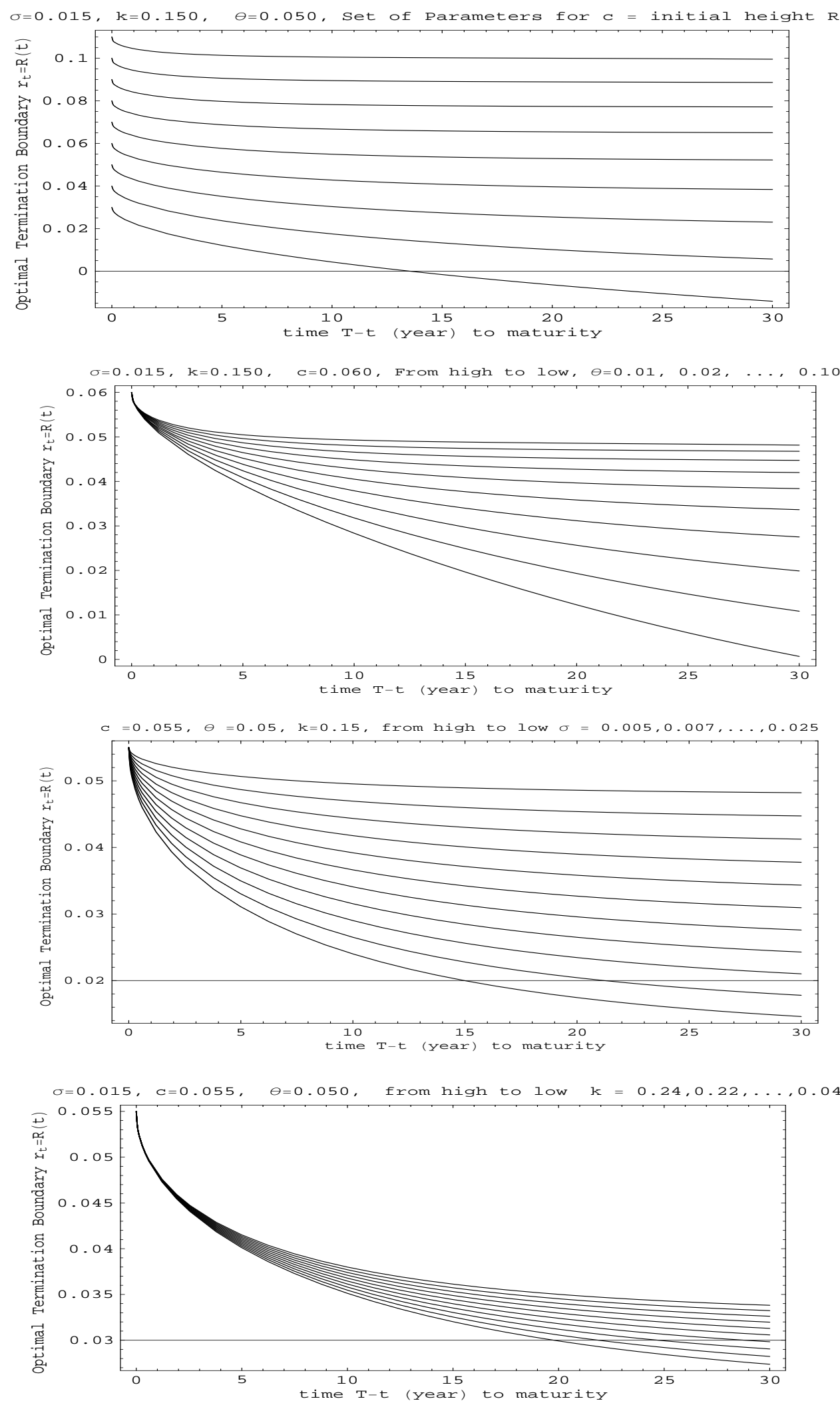

Figure 5. Optimal termination boundary $r_{t}=R(t)$ as function of time $T-t$ to maturity. Each set of curves corresponds to the changing of one parameter. 

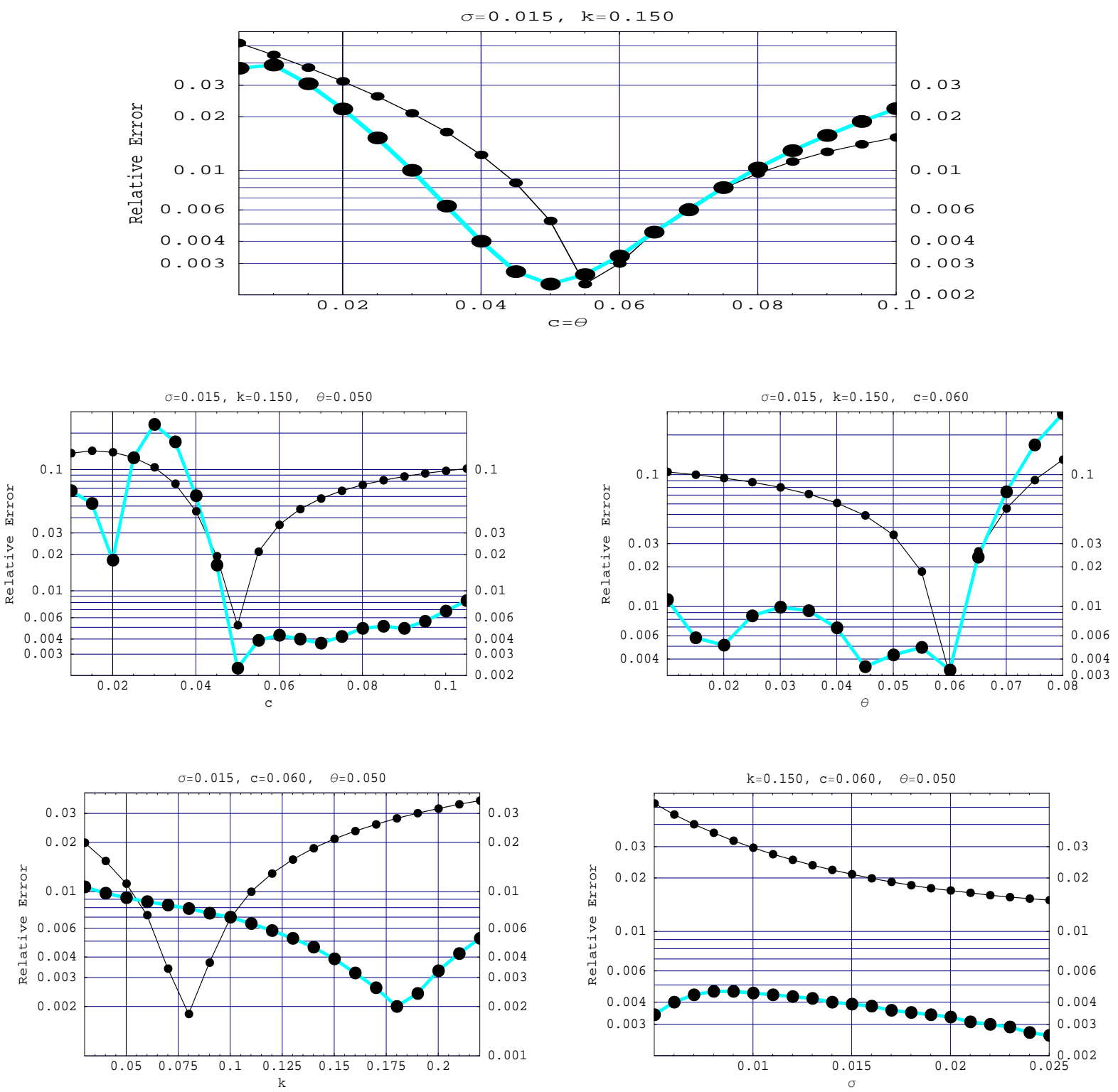

FiguRE 6. Relative errors of the first approximation $R_{I}$ (thin curve) and the second approximation $R_{I I}$ (thick curve) as functions of parameters. Relative errors are defined as

$$
\max _{t<T} \frac{\left|R(t)-R_{I}(t)\right|}{R(T)-R(-\infty)}, \quad \max _{t<T} \frac{\left|R_{(}(t)-R_{I I}(t)\right|}{R(T)-R(-\infty)}
$$

